\title{
Thermalization and many-body localization in systems under dynamic nuclear polarization
}

\author{
Andrea De Luca, ${ }^{1}$ Inés Rodríguez-Arias, ${ }^{1}$ Markus Müller, ${ }^{2,3,4}$ and Alberto Rosso ${ }^{1}$ \\ ${ }^{1}$ LPTMS, CNRS, Univ. Paris-Sud, Université Paris-Saclay, Orsay 91405, France \\ ${ }^{2}$ Condensed matter theory, Paul Scherrer Institute, CH-5232 Villigen PSI, Switzerland \\ ${ }^{3}$ The Abdus Salam International Centre for Theoretical Physics, Trieste 34151, Italy \\ ${ }^{4}$ Department of Physics, University of Basel, Klingelbergstrasse 82, CH-4056 Basel, Switzerland
}

(Received 1 March 2016; revised manuscript received 26 May 2016; published 6 July 2016)

\begin{abstract}
We study the role of dipolar interactions in the standard protocol used to achieve dynamic nuclear polarization (DNP). We point out that a critical strength of interactions is required to obtain significant nuclear hyperpolarization. Otherwise, the electron spins do not thermalize among each other, due to the incipient many-body localization transition in the electron spin system. Only when the interactions are sufficiently strong, in the so-called spin-temperature regime, they establish an effective thermodynamic behavior in the out-of-equilibrium stationary state. The highest polarization is reached at a point where the spin temperature is just not able to establish itself anymore. We provide numerical predictions for the level of nuclear hyperpolarization and present an analytical technique to estimate the spin temperature as a function of interaction strength and quenched disorder. We show that, at sufficiently strong coupling, nuclear spins perfectly equilibrate to the spin temperature that establishes among the spins of radicals.
\end{abstract}

DOI: 10.1103/PhysRevB.94.014203

\section{GENERAL INTRODUCTION}

The phenomenon of many-body localization is currently attracting a lot of attention, as it touches on various fundamental aspects of quantum statistical mechanics and quantum dynamics. However, despite its theoretical and conceptual appeal, very few consequences of practical relevance for physical processes are known so far. In this paper, we address a situation where localization does play an important role. We analyze the effects of incipient many-body localization in a system of driven quantum magnets, as standardly used in preparing polarized nuclear spins. Interestingly, we find that the achieved nuclear polarization is optimized by tuning parameters very close to the localization transition, approaching it from the delocalized side, thus implying a practical aspect of the localization transition.

The canonical formulation of quantum statistical mechanics assumes the contact between the system and an external reservoir. For a closed system, however, the description at large times using only few macroscopic parameters, such as the temperature or the chemical potential, implicitly assumes that the system itself acts as a thermal reservoir for its constituents. Establishing the validity of this assumption and understanding the regimes where it breaks down constitute the still open problem of quantum thermalization [1,2]. A simple way to probe thermalization is provided by quench protocols: a closed system is left to evolve starting from a nonthermal initial state, e.g., a state with a local excess of energy. In infinite, ergodic systems the excess energy spreads and dilutes indefinitely, so that any memory of the initial imbalance is lost. If only conserved quantities are retained from the initial state, one can argue that two eigenstates which are globally similar (e.g., have the same energy), cannot be distinguished by local measurements. This implies that expectation values and correlation functions of local observables in eigenstates must coincide with their values in the microcanonical ensemble, a statement which goes under the name of eigenstate thermalization hypothesis (ETH) [3].
However, exceptions from such thermalizing systems can occur when the considered systems are sufficiently disordered, which may induce ergodicity breaking [4,5] and the associated phenomenon of "many-body localization" (MBL) [6,7]. Recently, a variety of approaches based on perturbation theory [8,9], exact diagonalization [10], timedependent DMRG [11], renormalization group [12], local integrals of motion [13-15], and even rigorous mathematical results [16], provided independent indications of the existence of MBL phases. In this localized phase, eigenstates have a very different structure with low entanglement following an area law rather than a volume law [17]. In particular they do not obey the ETH, reflecting that the quantum dynamics is not ergodic anymore: Local expectation values exhibit strong fluctuations from eigenstate to eigenstate of the same energy density, since an extensive set of parameters $[15,18]$ is necessary to describe the long-time dynamics.

So far only few experimental indications of many body localization have been reported, in cold atoms [19] and trapped ions [20], where the difficulty of isolating quantum systems from a thermalizing bath can be overcome more easily than in solid matter which always hosts phonons. However, the possibility of hole burning in frustrated magnets such as $\mathrm{LiHo}_{x} \mathrm{Y}_{1-x} \mathrm{~F}_{4}$ and gadolinium gallium garnet [21] suggests that quantum magnets are promising solid state systems where localization phenomena might manifest themselves over very long time scales. After all, it was the apparent absence of spin diffusion in disordered magnets [22] that had led Anderson [6] to start the investigation of localization physics over half a century ago.

Being in a localized phase is often considered interesting for quantum technological applications, since entanglement is limited, or grows only very slowly in time [17,23,24]. In this paper, we show that being close to a localization transition is of great interest also in an entirely different domain, as it helps to achieve large nuclear hyperpolarization in quantum magnets.

In this paper, we study a class of quantum magnets relevant for so-called dynamic nuclear polarization (DNP). Those 
magnets generically host electron spins in a more or less random spatial configuration. To achieve a hyperpolarization the system is driven externally by microwaves. Apart from their immediate interest for DNP, these systems constitute an interesting example where the approach of a localized regime impacts the steady state of driven systems. This extends the scope of many-body localization physics beyond that of closed, time-independent problems. Note that in general, even in an ergodic regime with weak disorder, no simple thermodynamic description of a driven steady state can be expected. However, in the presence of weak driving and coupling with the outside world, the dephasing time of internal degrees of freedom is much faster than any other timescale, including the one associated with the driving. Hence the density matrix becomes essentially diagonal in the basis of the many-body eigenstates before the effect of the driving or the environment is felt. In this limit, if ETH holds for the isolated system, the driven state can nevertheless be described to a good approximation by the same set of intensive parameters as in equilibrium, however, with values that depend on the driving. In contrast, once localization occurs in the closed spin system, the driven steady state reflects the details of the local dynamics of the drive, such that simple equilibriumlike characterization will not emerge in general. The failure of an effective equilibrium description can serve as a fingerprint of localization physics.

In this paper we present clear signatures of thermalization and many-body localization in a model that describes the hyperpolarization of nuclear spins obtained via dynamic nuclear polarization (DNP) [25]. For a typical DNP procedure, one works with a compound doped with radicals (i.e., molecules with unpaired electrons), which is rapidly quenched to low temperatures to form a frozen, glassy matrix [26]. Empirically a glassy atomic structure is necessary to obtain significant hyperpolarization. The reason is not entirely established, but such a structure presumably helps to ensure a homogeneous dilution of radicals (and to prevent the clustering of radicals at lower-dimensional grain boundaries). Such a compound is then exposed to a strong magnetic field $B \simeq 3 \mathrm{~T}$, put in contact with a cold reservoir at $\beta^{-1} \simeq 1 \mathrm{~K}$ (see Fig. 1 left), and finally irradiated with microwaves which are resonant with the Zeeman splitting of a fraction of the radical spins. In the

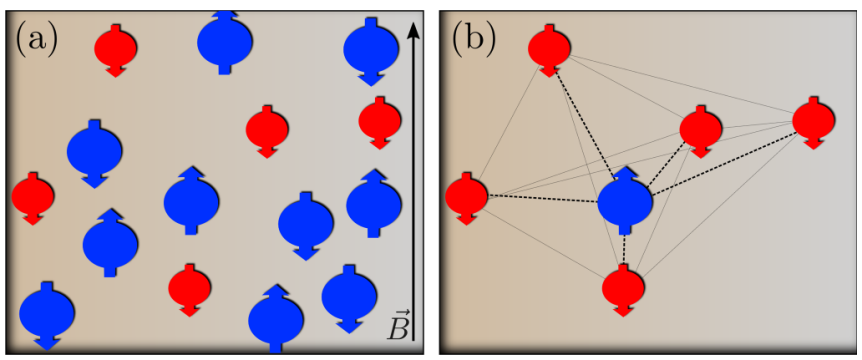

FIG. 1. Left: DNP system: the spatial positions of the nuclear spins of the compound (in blue) and the electron spins of few radical molecules (in red) are frozen in a glassy matrix. The spins are coupled via dipolar and hyperfine interactions. Right: The simplified model of Eq. (9). A single nuclear spin is surrounded by a collection of electron spins. Each spin is assumed to have random interactions with all others. The electronuclear couplings are much weaker than the dipolar couplings connecting the electron spins among each other. absence of microwave irradiation, the system reaches thermal equilibrium at the reservoir temperature $\beta^{-1}$ : The unpaired electrons are strongly polarized $(\sim 94 \%)$ while the nuclear spins are very weakly polarized $(<1 \%)$ as the nuclear Zeeman gap is about three orders of magnitude smaller than the level splitting of the electrons. In contrast, when the microwave frequency is close to the electron's Zeeman gap, the driven system of interacting electron and nuclear spins organizes into an out-of-equilibrium steady state with a huge nuclear polarization. The hyperpolarized sample can then be dissolved at room temperature [27], injected in patients, and used as metabolic tracer [28].

The traditional explanation of hyperpolarization goes back to the seventies and is based on the idea that the spin system, when irradiated, cools down to an effective thermodynamic state characterized by a low spin temperature [25,29]. This idea has been qualitatively confirmed in several experiments [30,31], where the polarizations $P_{n}$ of different nuclear species $\left({ }^{13} \mathrm{C},{ }^{15} \mathrm{~N},{ }^{89} \mathrm{Y}, \ldots\right)$ are simultaneously enhanced and well described by an equilibriumlike formula,

$$
P_{n}=\tanh \left(\beta_{s} \hbar \omega_{n} / 2\right),
$$

with a unique parameter $\beta_{s}$ (the inverse spin temperature) but different Zeeman gaps $\omega_{n}$ for the different species.

The success of the spin-temperature picture raises two fundamental questions:

(1) How can the emergence of an effective thermodynamical description be justified on a microscopic basis, and under what circumstances does an effective equilibrium indeed occur in the steady state?

(2) How to estimate and optimize $\beta_{s}$ as a function of the microscopic parameters which can be controlled in an experiment (e.g., radical concentration, magnetic field strength, microwave intensity, ... )?

As for the first question, we will see that an effective equilibrium does not emerge in just any spin system. Rather, the thermalization tendency in the closed spin system has to be sufficiently strong in order for an effective spin temperature to establish. This in turn poses constraints on the relative strength of disorder and interactions, which can be tuned independently in experiments, as we will discuss. Previous attempts to predict the value of $\beta_{s}$ were based on a purely phenomenological approach where an out-of-equilibrium quasithermal state was postulated from the outset, without questioning in which parameter regime the hypothesis was actually justified. In typical experimental settings, the spin temperature was estimated to be three orders of magnitude smaller than the lattice temperature. However, the theory predicted largely unrealistic levels of hyperpolarization: up to $80 \%$, as compared to the $20-40 \%$ observed in actual experiments [32].

In a recent work, we studied an ensemble of electron spins with random Zeeman gaps and subject to dipolar interactions, but with no nuclear spins [33]. Under microwave irradiation and assuming a weak coupling to the reservoir, we showed that the quantum dynamics can be reduced to a master equation for the occupation probabilities of the interacting eigenstates [34]. For $N=12$ spins, the (unique) stationary state can be extracted numerically from the master equation: The steady polarizations of the electrons reflect the ergodicity properties of eigenstates. In particular we have shown that 
an effective spin temperature emerges whenever: (i) ETH is satisfied by the isolated spin system, and (ii) dephasing inside the system happens on a fast timescale as compared to the driving and the relaxation processes involving the bath. In contrast, the signatures of a spin temperature disappear when the electron spins are many-body localized.

In this paper, we study numerically the hyperpolarization of a single nuclear spin interacting with all the electron spins (see Fig. 1 right). We show that its stationary polarization is consistent with the spin-temperature prediction of Eq. (1) when the spin Hamiltonian obeys ETH. This indicates that the steady-state parameter $\beta_{s}^{-1}$ behaves as a genuine temperature. Then, in the framework of the master equation already introduced in Ref. [33], we derive a technique to estimate $\beta_{s}$. The microscopic details of the system Hamiltonian are encoded only in the equilibrium spin-spin correlation function, which can be computed analytically. The results are in good agreement both with our numerical data and, qualitatively, with experimental measurements. This paves the way to finding a setup to optimize the hyperpolarization efficiency. We find that by decreasing the radical concentration, the spin temperature is lowered (and hence the hyperpolarization is increased) until the stationary nuclear polarization reaches a maximal value. Below this threshold concentration an approach postulating thermalization and an effective spin temperature would still predict a monotonous continuation of these trends. However, at this point the electron spins actually start to localize, thermalization is impeded, and as a consequence the average hyperpolarization becomes strongly suppressed, as it relies only on the excitation of local resonances. The maximum efficiency in hyperpolarization is thus a fingerprint of the incipient MBL transition, which occurs as the radical concentration is reduced or the external magnetic field is increased. Since both parameters can be controlled in standard DNP experiments, these theoretical predictions are subject to experimental verification.

The remainder of the paper is organized as follows: In Sec. II we introduce the DNP protocol and detail how we model it. In particular we discuss how the nuclear spin is coupled to the electron spins. In Sec. III, we show how the spin temperature can be defined for the stationary state and how its value can be computed numerically and analytically; in Sec. IV we present our results while Sec. V discusses the applicability and the breakdown of the spin-temperature ansatz as the closed system undergoes an MBL transition.

\section{THE DNP SETTING AND A SIMPLIFIED MODEL}

The enhancement of the nuclear polarization emerges in the framework of a correlated quantum spin system driven far from equilibrium by resonant microwave irradiation, while being in contact with the thermal reservoir of atomic lattice degrees of freedom, held at a temperature of typically about $T=1 \mathrm{~K}$. Describing the dynamical behavior of such a system is a formidable task, due to several competing interactions. Below, we specify the various ingredients and derive a simplified model that can be investigated both numerically and analytically.

\section{A. Description of the system}

In a DNP setup, there are three fundamental ingredients: (i) the internal spin dynamics governed by the competition between disorder and interactions; (ii) the microwave pumping; iii) (the weak contact with the reservoir. Let us describe each of them in turn.

The spin Hamiltonian. The spin system is composed of the electron spins $S_{i}(i=1, \ldots, N)$ associated with the radical molecules, and nuclear spins $I_{j}\left(j=1, \ldots, N_{n}\right)$ that one aims to hyperpolarize. Typically the concentration of the nuclei is about $N_{n} / N \approx 10^{3}$ times larger than that of the radicals. For simplicity we will consider both electron and nuclear spins to have spin $1 / 2$. All spins are exposed to a strong uniform magnetic field. The system Hamiltonian $\hat{H}_{\mathrm{S}}$ then takes the form

$$
\begin{aligned}
& \hat{H}_{\mathrm{S}}=\hat{H}_{Z}+\hat{H}_{\mathrm{int}}, \\
& \hat{H}_{Z}=\sum_{i=1}^{N}\left(\omega_{e}+\Delta_{i}\right) \hat{S}_{z}^{i}-\omega_{n} \sum_{j=1}^{N_{n}} \hat{I}_{z}^{j},
\end{aligned}
$$

where $\omega_{e}, \omega_{n}$ describe the average Zeeman energy of electron and nuclear spins in the external magnetic field, respectively. Because of $g$-factor anisotropies [25], the electronic Zeeman energies are subject to spatially fluctuating contributions $\Delta_{i}$, which constitute the dominant source of quenched disorder in the problem.

The interaction term $\hat{H}_{\text {int }}$ includes three contributions:

(1) the dipolar interaction between electron spins, of the form

$$
\hat{H}_{e-e}=\sum_{i<j} \frac{\mu_{0} \gamma_{e}^{2}}{4 \pi\left|\mathbf{r}_{i j}\right|^{3}}\left[\hat{\mathbf{S}}^{i} \cdot \hat{\mathbf{S}}^{j}-3\left(\hat{\mathbf{S}}^{i} \cdot \mathbf{n}_{i j}\right)\left(\hat{\mathbf{S}}^{j} \cdot \mathbf{n}_{i j}\right)\right],
$$

where $\mathbf{r}_{i j}$ is the distance vector between spins $i$ and $j, \mathbf{n}_{i j}=$ $\mathbf{r}_{i j} /\left|\mathbf{r}_{i j}\right|$, and $\gamma_{e}$ is the electron's gyromagnetic ratio. In a large magnetic field, this term can be regarded as a perturbation of the Zeeman energy described by (2). Therefore, hybridization between sectors of different total electronic magnetization $\hat{S}_{z}=\sum_{i} \hat{S}_{z}^{i}$ is strongly suppressed by the Zeeman gap. With a typical distance between radical molecules of $r_{e-e} \simeq 20 \AA$, the smallness of the parameter $\mu_{0} \gamma_{e}^{2} / r_{e-e}^{3} \omega_{e} \simeq 10^{-3}$ justifies the secular approximation [25], which projects the Hamiltonian onto subspaces of constant $\hat{S}_{z}$,

$$
\hat{H}_{e-e}=\sum_{i<j} U_{i j}\left[4 \hat{S}_{z}^{i} \hat{S}_{z}^{j}-\left(\hat{S}_{+}^{i} \hat{S}_{-}^{j}+\hat{S}_{-}^{i} \hat{S}_{+}^{j}\right)\right]
$$

with $U_{i j}=\mu_{0} \gamma_{e}^{2}\left(1-3 \cos ^{2} \theta_{i j}\right) /\left(16 \pi\left|\mathbf{r}_{i j}\right|^{3}\right)$. Here, $\theta_{i j}$ is the angle between the field along $z$ and $\mathbf{r}_{i j}$, and $\left[\hat{S}_{+}^{i}, \hat{S}_{-}^{i}\right]=2 \hat{S}_{z}^{i}$, with $\hat{S}_{ \pm}^{i}=\hat{S}_{x}^{i} \pm i \hat{S}_{y}^{i}$.

(2) the dipolar interaction between nuclear spins, which takes the same form as in (3) with $\gamma_{e} \rightarrow \gamma_{n} \simeq 10^{-3} \gamma_{e}$. These interactions are responsible for nuclear spin-diffusion [35], which tends to homogenize the polarization among the nuclear spins.

(3) the hyperfine interaction between the nuclear and the electron spins. For large Zeeman fields a projection onto 
$\hat{S}^{z}$-preserving terms yields

$$
\hat{H}_{e-n}=\sum_{i, j} D_{i j}^{(z)} \hat{S}_{z}^{i} \hat{I}_{z}^{j}+D_{i j}^{(x)} \hat{S}_{z}^{i} \hat{I}_{x}^{j}+D_{i j}^{(y)} \hat{S}_{z}^{i} \hat{I}_{y}^{j},
$$

where the hyperfine couplings take the form $D_{i j}^{(x, y)} \simeq$ $\mu_{0} \gamma_{e} \gamma_{n}\left|r_{e-n}^{-3}\right|$, with $r_{e-n}$ the distance between electrons and nuclei. Note that one can rotate each nuclear spin along the $z$ axis by an angle which depends on the configurations of the electrons and thereby achieve $D^{(y)}=0$. This comes at the price of introducing small, physically irrelevant phases to the transverse couplings, which we neglect. Here, given the small value of the hyperfine coupling $D_{i j}^{(x)} / \omega_{e} \simeq 10^{-8}-10^{-5}$, we retained again only the $\hat{S}^{z}$-preserving terms as already done for the dipolar interactions.

Coupling to the lattice. The bath modes must be included in the Hamiltonian $\hat{\mathcal{H}}$ of the full setup by adding a priori two terms

$$
\hat{\mathcal{H}}=\hat{H}_{\mathrm{S}}+\hat{H}_{\mathrm{R}}+\hat{H}_{\mathrm{S}-\mathrm{R}} .
$$

Here $\hat{H}_{\mathrm{R}}$ describes the dynamics of the bath, i.e., the motion of the atoms, while $\hat{H}_{\text {S-R }}$ captures the spin-bath interaction. The simplest description of such an interaction is obtained by assuming that each spin couples to the displacement of one individual vibrational mode localized close to it,

$$
\hat{H}_{\mathrm{S}-\mathrm{R}}=\lambda_{e} \sum_{\substack{i=1 \\ \alpha=x, y, z}}^{N} \hat{S}_{\alpha}^{i} \hat{\Phi}_{\alpha, e}^{i}+\lambda_{n} \sum_{\substack{j=1 \\ \alpha=x, y, z}}^{N} \hat{I}_{\alpha}^{j} \hat{\Phi}_{\alpha, n}^{j} .
$$

Here $\lambda_{e}, \lambda_{n}$ are the electron and nuclear spin-bath coupling constants, respectively. They fix the timescale of the relaxation processes. Since $\lambda_{n} \ll \lambda_{e}$, nuclear spin-bath coupling does not play any role in the hyperpolarization procedure. As usual the specific details of the bath Hamiltonian $\hat{H}_{\mathrm{R}}$ are unimportant, its main role being to maintain the bath at temperature $\beta^{-1} \equiv T$ and to quickly erase the memory of its past interactions with the system.

The microwave pumping. The microwave frequency $\omega_{\mathrm{MW}}$ is tuned close to the average electronic Zeeman energy $\omega_{e}$, so that it manages to flip electron spins occasionally, one at a time. This is described by the time-dependent Hamiltonian

$$
\hat{H}_{\mathrm{MW}}(t)=2 \omega_{1} \sum_{i} \hat{S}_{x}^{i} \cos \left(\omega_{\mathrm{MW}} t\right),
$$

where $\omega_{1}$ is the amplitude of the microwave field.

One of the central experimental observables is the so-called DNP profile, which describes the stationary value of nuclear polarization as a function of $\omega_{\mathrm{MW}}$ (see Fig. 3). In order to obtain a sizable enhancement over the thermal nuclear spin polarization, the microwave frequency $\omega_{\mathrm{MW}}$ must be chosen such as to lie within the range of the inhomogeneously broadened spectrum of Zeeman energies, $\left(\omega_{\mathrm{MW}}-\omega_{e}\right)^{2} \lesssim \overline{\Delta_{i}^{2}}$, so as to be resonant with a fraction of electron spin-flip transitions.

\section{B. Simplified model}

In this paper, we study a model of $N_{n}=1$ nuclear spin and $N$ interacting electron spins, in contact with a thermal reservoir and driven out-of-equilibrium by microwave irradiation. We limit the study to a single nuclear spin. This is motivated by the following experimental fact observed under standard DNP conditions: A sample of ${ }^{13} C$ pyruvic acid was doped with trytil radicals, a stable and very efficient polarizing agent. The choice of trytil is such that $\overline{\Delta_{i}^{2}} \ll \omega_{n}^{H}$, so that hydrogen is not DNP active. In this way, the only active nuclear species is ${ }^{13} \mathrm{C}$ and one observes that changing the nuclear spin concentration of ${ }^{13} C$ with respect to the spinless ${ }^{12} C$, does not affect the final polarization of ${ }^{13} \mathrm{C}$ as shown in [36]. This suggests that the enhancement in the nuclear polarizations is inherited from the steady state of the electrons and that different nuclei always have a homogeneous polarization: therefore, the study of a single nuclear spin should suffice to demonstrate the existence of a spin temperature and its transfer to the nuclear spins.

DNP is empirically found to be effective only in compounds where the atoms are frozen into a random, glassy configuration in which the distances $\mathbf{r}_{i j}$ between pairs of radicals are random. Since we will study relatively small systems, we model this in the limit of fully connected spin-spin interactions taking $U_{i j}$ as Gaussian random variables with zero mean and variance $U^{2} / N$. Note that the simplest choice of nonfluctuating couplings $U_{i j}=U / N$ would be pathological as it leads to the integrable Richardson model, which is always nonthermal [37]. Further, we neglect the Ising coupling $\hat{S}_{z}^{i} \hat{S}_{z}^{j}$ in (4), as it mostly modifies the instantaneous local fields seen by the various electron spins, but does not contribute in an essential way to the physics.

The inhomogeneous contributions to the Zeeman energy, $\Delta_{i}$, are taken equally spaced inside the interval $\left[-\Delta \omega_{e}, \Delta \omega_{e}\right]$ [38]. A more realistic approximation would consider a random distribution of the $\Delta_{i}$. However this choice would lead to strong finite size fluctuations, since in the small systems accessible by numerics, at most a single electron is in resonance with the microwave irradiation.

We finally arrive at the following simplified Hamiltonian:

$$
\begin{aligned}
\hat{H}_{S}= & \sum_{i=1}^{N}\left(\omega_{e}+\Delta_{i}\right) \hat{S}_{z}^{i}+\sum_{i<j} U_{i j}\left(\hat{S}_{+}^{i} \hat{S}_{-}^{j}+\hat{S}_{-}^{i} \hat{S}_{+}^{j}\right) \\
& -\omega_{n} \hat{I}_{z}+\sum_{i} D_{i} \hat{S}_{z}^{i} \hat{I}_{x} .
\end{aligned}
$$

Here, we neglect for simplicity the isotropic $z-z$ part in the hyperfine interaction and, as motivated above, only retain one representative term for the electron-nuclear spin coupling. This leads to quasithermalization of the nucleus by spin flips, if the electron spins establish a spin temperature. The $D_{i}$ 's are drawn from a normal distribution of zero average and variance $D^{2} / N$. The strength $D$ is chosen so as to ensure sufficient coupling to the electrons without causing any significant perturbation of their state. The nuclear spin thus acts effectively as a thermometer.

Let us start by considering the relaxation dynamics in the absence of microwaves. In order to treat the interaction with the reservoir, we employ a significant separation of timescales in our problem. As we observed in the previous section, the leading term in the Hamiltonian is proportional to $\omega_{e} \simeq 100 \mathrm{GHz}$ in typical magnetic fields. The order of magnitude of the remaining terms of the spin Hamiltonian 
$\left(\Delta \omega_{e}, U, D, \omega_{n}\right)$ range from a few to $100 \mathrm{MHz}$. On the other hand, the rate of energy exchange with the bath can be estimated by the inverse of the electronic relaxation time, $1 / T_{1 e}$, which is of the order of $1 \mathrm{~Hz}$ [39]: This implies a weak coupling between electron spins and bath modes. In this limit, it is possible to derive, within the Born-Markov approximation scheme, an evolution equation for the density matrix in Lindblad form. This approach is analogous to the equations derived in Ref. [34] and has the advantage of being directly connected to the microscopic model [40]. The details of this derivation were presented in Ref. [33]. Summarizing, the resulting evolution of the density matrix of the spin system $\rho$ is governed by the Lindblad equation

$$
\frac{d \rho}{d t}=-i\left[\hat{H}_{S}, \rho\right]+\mathcal{L}[\rho] .
$$

The last term contains the nonunitary dynamics with two kinds of contributions: (i) electron spin-flip processes [due to the coupling of bath modes with $S_{x}^{i}, S_{y}^{i}$ in (7)] which induce transitions between pairs of eigenstates of $\hat{H}_{S}$ and involve an exchange of energy $\simeq \omega_{e}$ with the lattice; (ii) processes due to the coupling of the bath modes with $\hat{S}_{z}^{i}$ or $\hat{I}_{z}$ for which the exchange of energy vanishes in the noninteracting limit. The latter thus contributes mostly to the dephasing of off-diagonal elements of the density matrix in the basis of eigenstates of $\hat{H}_{S}$, while the former dominates the relaxation of the diagonal elements, so that at long times the density matrix reaches the thermal state with the lattice temperature: $\rho=Z^{-1} e^{-\beta \hat{H}_{S}}$.

The precise estimation of the different timescales is difficult. Experimentally, one knows that $T_{1 e} \simeq 1 \mathrm{~s}$ and $T_{2 e} \simeq$ $10^{-6} \mathrm{~s}$, associated with the relaxation of the longitudinal and the transverse spin polarization, respectively [41]. Given the huge difference between the two timescales [42], we assumed in Ref. [33] that the quantum dynamics of the system can be reduced from the Lindblad equation to a classical master equation for the occupation probabilities $p_{n}$ of the many-body eigenstates $\Psi_{n}$

$$
\frac{d p_{n}}{d t}=\sum_{n^{\prime} \neq n} W_{n \rightarrow n^{\prime}}^{\mathrm{bath}} p_{n^{\prime}}-W_{n \rightarrow n^{\prime}}^{\mathrm{bath}} p_{n},
$$

where the transition rates have the form:

$$
W_{n \rightarrow n^{\prime}}^{\text {bath }}=\frac{2 h_{\beta}\left(\Delta \epsilon_{n, n^{\prime}}\right)}{T_{1 e}} \sum_{j=1}^{N} \sum_{\alpha=x, y, z}\left|\left\langle n\left|\hat{S}_{\alpha}^{j}\right| n^{\prime}\right\rangle\right|^{2},
$$

with $\Delta \epsilon_{n, n^{\prime}}=\epsilon_{n}-\epsilon_{n^{\prime}}$. Equation (12) describes spin flips induced by the interaction with the external bath on a timescale $T_{1 e}$ and the function $h_{\beta}(x)=e^{\beta x} /\left(1+e^{\beta x}\right)$ assures detailed balance and convergence to Gibbs equilibrium at temperature $\beta^{-1} \equiv T \simeq 1 \mathrm{~K}$. The coupling of the nuclear spin to the bath modes induces similar transitions but with a much smaller rate, since $T_{1 n} \gtrsim 10^{3} T_{1 e}$.

We now include the coupling to the microwave radiation as described by Eq. (8). As the microwave field is time dependent, its treatment requires some care. A precise estimation of the microwave amplitude $\omega_{1}$ is difficult, since it is hard to evaluate the fraction of emitted power which actually reaches the sample in a given experiment. In general, we can assume that $\omega_{1}$ is within the range of tens to hundreds of $\mathrm{kHz}$. Thus, the transition rate satisfies $\omega_{1}^{2} T_{2 e} \ll \omega_{e}$. Together with the condition $\Delta \omega_{e} \ll \omega_{e}$ this ensures that we can safely employ the rotating-wave approximation: It entails transforming the Hamiltonian and the density matrix into a rotating frame, i.e.,

$$
\begin{gathered}
\rho^{(r)}=e^{i \hat{S}_{z} \omega_{\mathrm{MW}} t} \rho e^{-i \hat{S}_{z} \omega_{\mathrm{MW}} t}, \\
\operatorname{Tr}[\hat{O} \rho]=\operatorname{Tr}\left[e^{-i \hat{S}_{z} \omega_{\mathrm{MW}} t} \hat{O} e^{i \hat{S}_{z} \omega_{\mathrm{MW}} t} \rho^{(r)}\right],
\end{gathered}
$$

where the last line holds for any observable $\hat{O}$, and $\hat{S}_{\alpha}=$ $\sum_{i} \hat{S}_{\alpha}^{i}$. For observables that commute with $\hat{S}_{z}$, such as the individual polarizations $\hat{S}_{z}^{i}$, expectation values can safely be computed in the rotating frame. The advantage of the transformation (13) is that, since $\left[\hat{S}_{z}, \hat{H}_{S}\right]=0$, the evolution of $\rho^{(r)}$, in the presence of microwaves, is the same as that for $\rho$ in (10), where, apart from rapidly oscillating terms, the Hamiltonian has been replaced by the time independent

$$
\hat{H}_{S}^{\text {rot }} \rightarrow \hat{H}_{S}-\omega_{\mathrm{MW}} \hat{S}_{z}+\omega_{1} \hat{S}_{x},
$$

while $\mathcal{L}[\rho] \rightarrow \mathcal{L}\left[\rho^{(r)}\right]$. Within this approximation the effect of microwaves can be again treated as a master equation

$$
\frac{d p_{n}}{d t}=\sum_{n^{\prime} \neq n} W_{n \rightarrow n^{\prime}} p_{n^{\prime}}-W_{n \rightarrow n^{\prime}} p_{n},
$$

where the rate has now the form $W_{n \rightarrow n^{\prime}}=W_{n \rightarrow n^{\prime}}^{\mathrm{bath}}+W_{n \rightarrow n^{\prime}}^{\mathrm{MW}}$, with

$$
W_{n \rightarrow n^{\prime}}^{\mathrm{MW}}=\frac{4 \omega_{1}^{2} T_{2 e}^{\mathrm{bath}}\left|\left\langle n\left|\hat{S}_{x}\right| n^{\prime}\right\rangle\right|^{2}}{1+\left(T_{2 e}^{\mathrm{bath}}\right)^{2}\left(\left|\epsilon_{n}-\epsilon_{n^{\prime}}\right|-\omega_{\mathrm{MW}}\right)^{2}} .
$$

The use of the master equation is justified as long as $\omega_{1}^{2} T_{2 e}^{\text {bath }}$ is small as compared to the amplitude of the intraspin interaction terms.

In this formula, $T_{2 e}^{\text {bath }}$ is the dephasing time of eigenstates due to the coupling to a bath. This parameter is hard to access experimentally. Indeed, a single electron spin dephases even in the absence of the external reservoir due to dipolar coupling with the other spins. In particular, in the ergodic phase, the system acts as its own reservoir and there is an internal notion of dephasing. The experimentally measured $T_{2 e}$, typically of order $10^{-6} \mathrm{~s}$, is a result of both the dephasing due to the bath and due to dipolar couplings. We nevertheless take it as an estimate (lower bound) for the bath-induced part of the dephasing time. We then treat $T_{2 e}^{\text {bath }}$ as a separate input parameter independent of the interaction strength $U$. We emphasize that for $\omega_{1} \neq 0$, the rates $W_{n \rightarrow n^{\prime}}$ do not respect a detailed balance condition and thus, the stationary state will be out-ofequilibrium.

\section{THE SPIN-TEMPERATURE ANSATZ FOR THE STATIONARY STATE}

In this section we discuss the behavior of the system ignoring the nuclear spin, which is weakly coupled and serves only as a thermometer, without acting back on the electronic system. The time evolution of the spin system can be decomposed into two regimes. Occasionally, the thermal reservoir or the microwave field flips a single electron spin. Subsequently, fast dephasing brings the system essentially into a classical mixture of eigenstates of $\hat{H}_{\mathrm{S}}$ (this is a good 
description as far as local observables are concerned). Since the spin flip is a perturbation localized in space, it is natural to ask how much information about the position of the flipped spin is retained after dephasing. As long as the electron spins form an ergodic system no local information except for the increment of the conserved quantities (energy and electron spin polarization) will remain. It implies that after the typical dephasing time, for any local observable, the expectation value on the projected state coincides with the average over all states characterized by the same value of energy and electron polarization. In a canonical description this corresponds to the equilibrium average in the presence of two intensive parameters:

$$
p_{n} \simeq p_{n}^{\mathrm{Ans}}=Z^{-1} e^{-\beta_{s}\left(\epsilon_{n}+h s_{z, n}\right)},
$$

where $Z$ is fixed by normalization. The inverse spin temperature $\beta_{s}$ is the parameter conjugate to the energy, while the effective magnetic field $h$ is conjugate to the electron magnetization: $\epsilon_{n}$ and $s_{z, n}$ are the eigenvalues of $\hat{H}_{\mathrm{S}}$ and $\hat{S}_{z}$ on $|n\rangle$ in the laboratory (nonrotating) frame. In the following we compute both parameters following two complementary approaches:

(1) A fitting method (FM), which allows us to obtain estimates $\left(\beta_{s}^{\mathrm{FM}}, h^{\mathrm{FM}}\right)$ of the two intensive parameters $\left(\beta_{s}, h\right)$. This is done by imposing that the distribution $p_{n}^{\text {Ans in (18) has }}$ the same average energy and electron magnetization as found in the numerical simulations. This is the maximum entropy state given that these locally conserved quantities have become stationary.

(2) A perturbative expansion (PE) for weak $U$ and for an infinite number of spins interacting via (9). This method is based on the observation that the variation of energy and electron polarization, induced by single spin flip transitions, are encoded in the spin-spin correlation functions computed with the density matrix at time $t$. Using (18), these functions can be computed, order by order in perturbation theory in $U$. The values of $\beta_{s}$ and $h$ can then be estimated (and denoted by $\beta_{s}^{\mathrm{PE}}$ and $h^{\mathrm{PE}}$ ) imposing a balance for the total in- and outflow of energy and electron spin polarization due to interactions with the radiation and the bath.

We now explain the details of the two procedures.

\section{A. Fitting method}

Our fitting method allows us to fix the parameters of the spin-temperature ansatz for a given value of $U$. We use a numerical simulation to obtain the stationary state of Eq. (16) in a given realization of the couplings $U_{i j}$ and $D_{i}$. First, by exact diagonalization of the Hamiltonian $\hat{H}_{\mathrm{S}}$, we compute the $2^{N+1}$ eigenstates $|n\rangle$. The exponential growth of the Hilbert space strongly limits the accessible sizes, and thus we restrict ourselves to $N=12$. The rates in Eqs. $(12,17)$ can be computed exactly as matrix elements between pairs of eigenstates. Then, the occupation probabilities in the stationary state $p_{n}^{\text {stat }}$ are obtained by setting $d p_{n} / d t=0$ in Eq. (16) and solving the resulting linear system. Typically there is a unique solution to these equations, both in the ergodic $\left(U / \Delta \omega_{e} \gg 1\right)$ and the many-body localized $\left(U / \Delta \omega_{e} \ll 1\right)$ phases. Unlike in the dynamics of closed systems, there is no memory of the initial state, even in the many-body localized phase. The contact with the reservoir and the microwaves renders the stationary state memoryless, even though strongly nonthermal.

Under the hypothesis of Eq. (18) for ergodic phases, a natural way to determine the two parameters $\beta_{s}$ and $h$ consists in matching the expectation values of the two conserved quantities, i.e., requiring

$$
\begin{aligned}
{\overline{\left\langle\hat{H}_{\mathrm{S}}\right\rangle_{\mathrm{stat}}}}={\overline{\left\langle\hat{H}_{\mathrm{S}}\right\rangle_{\mathrm{Ans}}}}, \\
\overline{\left\langle\hat{S}_{z}\right\rangle_{\mathrm{stat}}}=\overline{\left\langle\hat{S}_{z}\right\rangle_{\mathrm{Ans}} .}
\end{aligned}
$$

Here $\langle\hat{O}\rangle_{\text {stat }}=\sum_{n} p_{n}^{\text {stat }}\langle n|O| n\rangle$ and similarly $\langle O\rangle_{\text {Ans }}=$ $\sum_{n} p_{n}^{\text {Ans }}\langle n|O| n\rangle$, and the overline represents the average over different realizations of dipolar $\left(U_{i j}\right)$ and hyperfine $\left(D_{i}\right)$ couplings, while the Zeeman splittings are always taken to be equally spaced inside the interval $\left[-\Delta \omega_{e}, \Delta \omega_{e}\right]$. In Eq. (19), the values of $s_{z, n}, \epsilon_{n}$ and $p_{n}^{\text {stat }}$ are obtained numerically for each realization. The parameters $\beta_{s}^{\mathrm{FM}}$ and $h^{\mathrm{FM}}$ are then determined as the (realization-independent) values which solve Eqs. (19).

\section{B. Perturbative expansion for weak interactions}

A different estimation of the quasiequilibrium parameters is based on the time evolution of the total energy and magnetization. Indeed, since these two quantities are conserved by $\hat{H}_{S}$, their values merely change due to the spin-flip transitions induced by the reservoir and the microwave field. All the microscopic details are then encoded in the spinspin correlation function, that at large times, writes as (see Appendix A)

$$
\chi_{i j}(u, v)=\sum_{n} p_{n}^{\text {stat }}\left\langle n\left|U(u, v) \hat{S}_{x}^{i} U^{\dagger}(u, v) \hat{S}_{x}^{j}\right| n\right\rangle
$$

with $U(u, v)=e^{i\left(\hat{H}_{S} u+\hat{S}_{z} v\right)}$. If the spin-temperature ansatz (18) holds, $\chi_{i j}(u, v)$ reduces to the calculation of the spin-spin correlation function at equilibrium (see Appendix B). Since, at stationarity, the total exchange of magnetization and energy must vanish, we obtain two conditions, which in the limit of negligible interaction strength $U$ can be written explicitly as (see Appendix C)

$$
\begin{aligned}
& \int d \omega f(\omega) \kappa(\omega)=0, \\
& \int d \omega \omega f(\omega) \kappa(\omega)=0 .
\end{aligned}
$$

Hereby $f(\omega)=\frac{1}{N} \sum_{i} \delta\left(\omega_{e}+\Delta_{i}-\omega\right)$ is the distribution of the Zeeman energies of the electron spins, which we, at large $N$, choose to be uniform in $\left[\omega_{e}-\Delta \omega_{e}, \omega_{e}+\Delta \omega_{e}\right]$. Setting $P_{e}\left(\omega_{e}+\Delta_{i}\right)=\left\langle\hat{S}_{z}^{i}\right\rangle_{\text {stat }}$, computed at $U=0$, the function $\kappa(\omega) \equiv \frac{d P_{e}(\omega)}{d t}$ describes the rate of change of polarization (due to radiation and reservoir) of the spins with Zeeman energy $\omega$, i.e.,

$$
\kappa(\omega)=\frac{P_{0}(\omega)-P_{e}(\omega)}{2 T_{1 e}}-\frac{T_{2 e}^{\text {bath }} \omega_{1}^{2} P_{e}(\omega)}{\left(T_{2 e}^{\text {bath }}\right)^{2}\left(\omega-\omega_{\mathrm{MW}}\right)^{2}+1},
$$

where $P_{0}(\omega)=-\tanh (\beta \omega / 2)$ is the equilibrium polarization in the absence of microwaves. 
In the absence of interactions the electron polarization $P_{e}(\omega)$ is fixed by the condition $\kappa(\omega)=0$, since different frequencies do not mix. In contrast, the spin-temperature ansatz implies that $P_{e}(\omega)=-\tanh \left(\beta_{s}(\omega+h) / 2\right)$ in the steady state: The resulting nonzero value of $\kappa$ at most frequencies is then compensated by the interaction-mediated redistribution of the conserved quantities among the spins. In Eq. (22), the first term accounts for the relaxation of polarization due to the reservoir, while the second term captures the effect of the microwave field. A very similar form of $\kappa$, which we re-derive for completeness in Appendix D, was proposed by Borghini [32]:

$$
\kappa_{\mathrm{Borg}}(\omega)=\frac{P_{0}(\omega)-P_{e}(\omega)}{2 T_{1 e}}-\pi \omega_{1}^{2} P_{e}(\omega) \delta\left(\omega-\omega_{\mathrm{MW}}\right),
$$

in which the Lorentzian absorption in (22) was replaced with an infinitely sharp $\delta$ function, assuming that only electron spins in exact resonance with $\omega_{\mathrm{MW}}$ are flipped by the microwaves. However, this postulate leads to a substantial underestimation of the spin temperature $[43,44]$ as compared to experimentally observed values. In Appendix D, we recall the results of the Borghini model, discuss its main limitations, and show how to improve on it to capture the most important effect of interactions.

Using the expression (22) instead yields values closer to experimental observations for $\beta_{s}^{\mathrm{PE}}, h^{\mathrm{PE}}$. Moreover, we can extend Eq. (21) to finite $U$ and take into account perturbative corrections for $U \ll \Delta \omega_{e}$. They lead to modified stationarity conditions (see Appendix C):

$$
\begin{aligned}
& \int d \omega f(\omega)\left[\kappa(\omega)+\frac{U^{2}}{2} \int d \omega^{\prime} f\left(\omega^{\prime}\right) \kappa_{1}\left(\omega, \omega^{\prime}\right)\right]=0, \\
& \int d \omega f(\omega)\left[\omega \kappa(\omega)+\frac{U^{2}}{2} \int d \omega^{\prime} f\left(\omega^{\prime}\right) \kappa_{2}\left(\omega, \omega^{\prime}\right)\right]=0,
\end{aligned}
$$

where the second-order corrections are given by

$$
\begin{gathered}
\kappa_{1}\left(\omega, \omega^{\prime}\right)=\frac{d}{d \omega}\left(\frac{\kappa(\omega)-\kappa\left(\omega^{\prime}\right)}{\omega-\omega^{\prime}}\right), \\
\kappa_{2}\left(\omega, \omega^{\prime}\right)=\frac{d}{d \omega}\left(\frac{\omega \kappa(\omega)-\omega^{\prime} \kappa\left(\omega^{\prime}\right)}{\omega-\omega^{\prime}}\right) .
\end{gathered}
$$

A more phenomenological, but efficient way of accounting for interaction effects consists of using Eqs. (21) and interpreting dephasing time $T_{2 e}$ as that of single spins (instead of that of eigenstates, as within the Lindblad equation). It is natural to assume the dephasing rate to be a direct sum of rates due to the bath and due to dipolar interactions,

$$
\frac{1}{T_{2 e}(U)}=\frac{1}{T_{2 e}^{\text {bath }}}+C \frac{U^{2}}{\Delta \omega_{e}} .
$$

The second term is the form expected from an estimate based on Fermi's golden rule applied to spin flip processes among the electron spins, with a numerical constant $C=O(1)$. This phenomenological approach only captures the most obvious interaction effect. However, it neglects, e.g., the $\omega$ dependence of the resulting $T_{2 e}(U)$ or self-energy effects, that modify the spectrum. All of those refinements are in principle contained in the microscopic and exact, but less intuitive description (24).
TABLE I. Summary of the parameters used in our calculations.

\begin{tabular}{lcccc}
\hline \hline$T_{1 e}$ & $T_{2 e}^{\text {bath }}$ & $B$ & $\beta$ & $U$ \\
\hline $1 . \mathrm{s}$ & $10^{-6} \mathrm{~s}$ & 3.35 Tesla & $0.83 \mathrm{~K}^{-1}$ & $2.0 \div 45.02 \pi \mathrm{MHz}$ \\
& $\omega_{e}$ & $\Delta \omega_{e}$ & $\omega_{1}$ & $\omega_{n}$ \\
\hline & $93.92 \pi \mathrm{GHz}$ & $542 \pi \mathrm{MHz}$ & $252 \pi \mathrm{kHz}$ & $202 \pi \mathrm{MHz}$ \\
\hline \hline
\end{tabular}

As we show in Appendix D (Fig. 6), this improved phenomenological model reproduces very successfully the actual value and interaction dependence of the spin temperature, and hence, of the nuclear polarization.

\section{NUMERICAL RESULTS}

We focus on the standard conditions of DNP experiments using trytils for the hyperpolarization of ${ }^{13} C$ nuclear spins. The values of the microscopic parameters are taken from actual experiments and are summarized in Table I.

The aim of this section is twofold: On one hand, we test the range of validity of the spin-temperature assumption for the stationary state; on the other hand, we quantify the finite-size correction affecting our numerical results for $N=$ 12 (averaged over at least 100 configurations). To achieve this, we focus on the stationary nuclear spin polarization. The nuclear spin is weakly coupled to the electron spins and acts simply as a thermometer; within the spin-temperature ansatz, its stationary polarization is therefore expected to take the form of Eq. (1). We can then compare two different estimations for $P_{n}$ :

(1) the value predicted by Eq. (1), with $\beta_{s}=\beta_{s}^{\mathrm{FM}}$ as obtained from the fitting method explained in Sec. III A;

(2) the value predicted by Eq. (1), with $\beta_{s}=\beta_{s}^{\mathrm{PE}}$, obtained from the perturbative expansion in the thermodynamic limit $N \rightarrow \infty$ of the fully-connected model, as explained in Sec. III B;

with the exact value obtained upon averaging over several realizations the stationary nuclear spin polarization obtained from the numerical procedure over several realizations

$$
P_{n}=\overline{\sum_{m} p_{m}^{\text {stat }}\left\langle m\left|I_{z}\right| m\right\rangle} .
$$

In Fig. 2 the dashed lines are the prediction for the nuclear polarization with the analytical estimates for the spin temperature. For small interaction, the perturbative results of Eqs. (24) are in good agreement with the numerical data. Upon increasing $U$, the lowest order result Eq. (24) cannot be expected to be accurate anymore, but it still correctly describes the decrease in polarization, and thus captures the important, but hitherto unexplained, effect of radical concentration seen in the experiments [27,36]: As the radical concentration, and thus $U$, is increased, the nuclear polarization decreases.

On the left of Fig. 2, we show the behavior of the nuclear hyperpolarization as a function of the dipolar coupling $U$, at fixed disorder strength. We observe two regimes: In the strongly-interacting regime, the concept of a spin-temperature perfectly applies to the stationary states and the polarization of the nuclear spin collapses with the prediction given by (1) and 

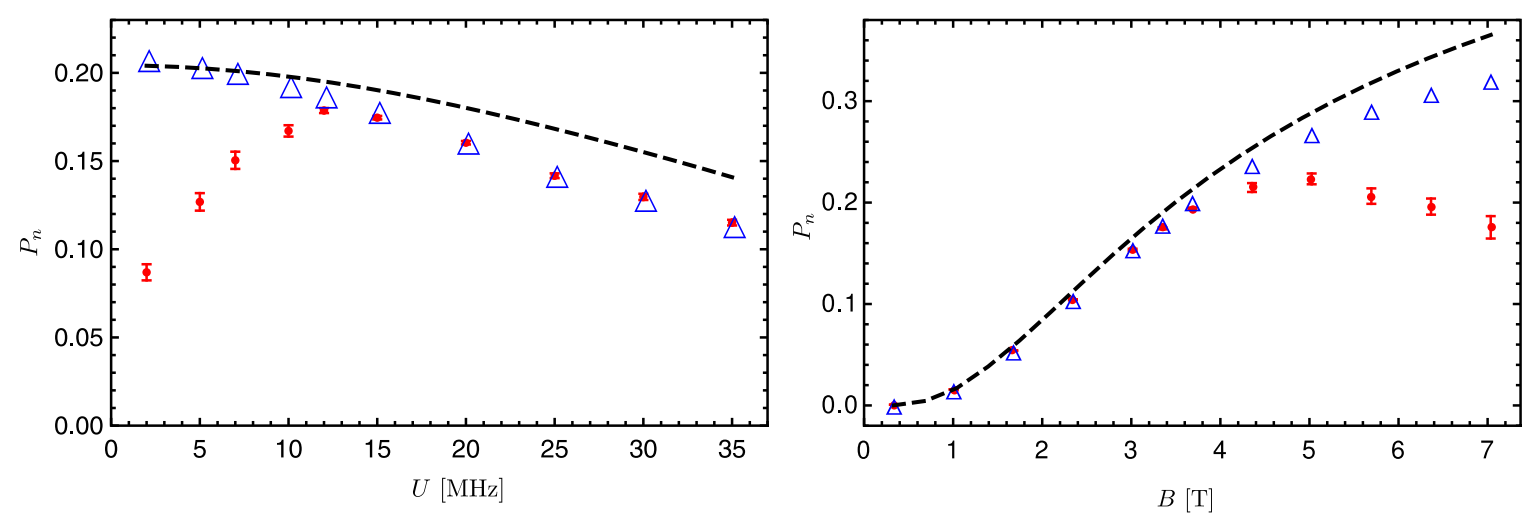

FIG. 2. Breakdown of the existence of a spin temperature. The steady state polarization of the nuclear spin is plotted as a function of the typical dipolar coupling strength $U$ at a fixed magnetic field $B=3.35 \mathrm{~T}$ (left) and as a function of the magnetic field $B$ at fixed $U=15 \mathrm{MHz}$ (right). The red circles show the nuclear polarization in the stationary state averaged over many realizations (the error bar shows the standard deviation). These results are compared with values of the nuclear polarization obtained from Eq. (1). The blue triangles correspond to the spin temperature, $\beta_{s}^{-1}$ estimated by the fitting method of (19), while the black dashed line corresponds to the perturbative expansion discussed in Sec. III B. Both plots show the breakdown of the spin-temperature assumption once the spread of Zeeman inhomogeneities dominates over the strength of the dipolar interactions.

$\beta_{s}=\beta_{s}^{\mathrm{FM}}$. For $U \lesssim 10 \mathrm{MHz},\left(U / \Delta \omega_{e} \lesssim 0.2\right)$ the observed polarization is much smaller than the value expected by postulating a spin-temperature state of the electrons. This shows that at least for our finite $N$ simulations, the thermal regime breaks down. We expect that this behavior remains true in the thermodynamic limit of systems with a finite connectivity among electron spins. A similar behavior is observed in the right panel of Fig. 2, where the magnetic field is varied at constant interaction strength $U$. We recall that the disorder strength, i.e., the spread of inhomogeneous contributions to the Zeeman energies, $\Delta \omega_{e}$, increases proportionally with the strength of the external magnetic field $B$. Thus, at small fields the dominating interactions establish a spin temperature, whereas at large fields, the internal thermalization of the electron system breaks down. We note that both methods

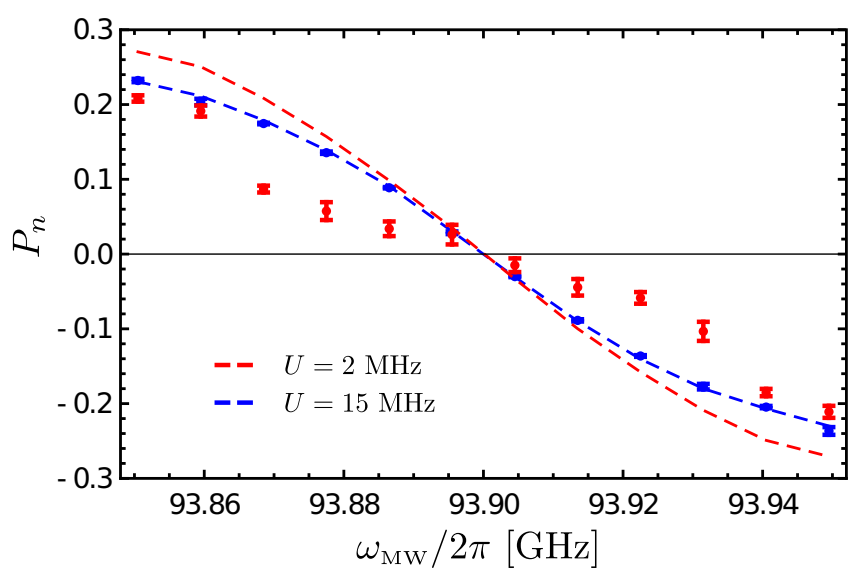

FIG. 3. The DNP profile, i.e., the steady state value of the nuclear polarization as a function of the microwave frequency. In red we show the results for $U=2 \mathrm{MHz}$, in blue the results for $U=15 \mathrm{MHz}$. Symbols correspond to the steady state value of the nuclear polarization, while the dashed line corresponds to Eq. (1) with the spin temperature $\beta_{s}^{-1}$ obtained with the fitting method. to compute the spin temperature-the fitting procedure for $N=12$ systems and the perturbative analytical calculation for a mean field $(N=\infty)$ system-yield compatible results. Their difference seems to stem mostly from the error due to the restriction of the perturbative calculation to quadratic order in $U$, rather than due to the effects of comparing $N=12$ with the mean field limit $N=\infty$.

In Fig. 3, we show an important characteristic of a DNP experiment, known as the DNP profile: the hyperpolarization $P_{n}$ as a function of the irradiated microwave frequency $\omega_{\mathrm{MW}}$. We compare the exact value of $P_{n}$ from (28) with the prediction of (1) into which we plug $\beta^{\mathrm{FM}}$ obtained from the fitting method. For $U=15 \mathrm{MHz}\left(U / \Delta \omega_{e} \simeq 0.3\right)$, the two results are consistent, while for $U=2 \mathrm{MHz}\left(U / \Delta \omega_{e} \simeq 0.04\right)$, we generally observe a small hyperpolarization, with the exception of a window of width $O\left(\omega_{n}\right)$ around $\omega_{\mathrm{MW}}=\omega_{e} \pm \Delta \omega_{e}$, where the polarization is induced by the so-called solid effect [45]. The latter consists of the following: Even in the absence of dipolar interactions among the electrons, the presence of hyperfine interactions allows the microwaves to excite a prohibited transition, where the nuclear spin is flipped together with an electron with a transition frequency $\omega_{i}=\omega_{\mathrm{MW}} \pm \omega_{n}$. This effect is more prominent at the boundaries of the microwave spectrum of interest, $\omega_{\mathrm{MW}} \in\left[\omega_{e}-\Delta \omega_{e}, \omega_{e}+\Delta \omega_{e}\right]$. Indeed, in the bulk of the spectrum the two types of simultaneous transitions of an electron and a nuclear spin have almost equal probability, and therefore their effects tend to cancel.

\section{DISCUSSION}

The data presented in Fig. 2 indicate a marked difference from thermal behavior, as the dipolar couplings become weaker (i.e., for low radical concentration) and/or by increasing magnetic fields. Since the hyperfine interaction between the nuclei and the electrons is left unchanged in both these cases, this phenomenology actually reflects a change in the electron system. Indeed, in order for the electron spins to act as a bath for the nucleus, they have to be in a "thermal 

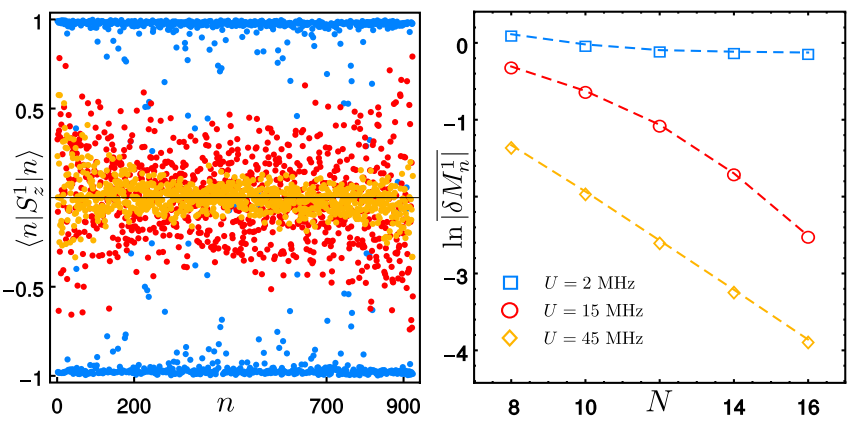

FIG. 4. Numerical investigation of the ETH hypothesis for a closed system of $N$ electron spins. (a) Average polarization of the selected spin $\hat{S}_{z}^{1}$ in the eigenstates $|n\rangle$ of $N=12$ electron spins with vanishing total magnetization. The eigenstates are ordered according to increasing energy. Data are shown for three different values of the dipolar strength: $U=2 \mathrm{MHz}$ (blue), $15 \mathrm{MHz}$ (red), $45 \mathrm{MHz}$ (blue); $\Delta \omega_{e}=54 \mathrm{MHz}$. (b) Difference in local magnetization between consecutive eigenstates, $\delta M_{n}^{1}=\left\langle n+1\left|\hat{S}_{z}^{1}\right| n+1\right\rangle-\left\langle n\left|\hat{S}_{z}^{1}\right| n\right\rangle$, averaged over disorder realizations and globally unpolarized eigenstates, for different system sizes $N=8, \ldots, 16$. An exponential decay of $\overline{\delta M_{n}^{1}}$ with $N$ indicates a thermal phase obeying ETH, while a saturation signals many-body localization.

phase." Upon decreasing the value of $U$, the disorder in the inhomogeneous Zeeman energies, $\Delta \omega_{e}$, becomes dominant: the eigenstates fail to be ergodic and enter a many-body localized phase. Indications of this transition are shown in Fig. 4 where we study the expectation value of the polarization of a selected electron spin, say $S^{1}$, on all eigenstates within the sector of vanishing total electron polarization: $\left\langle n\left|\hat{S}_{z}^{1}\right| n\right\rangle$. The left of Fig. 4 visualizes the qualitatively different behaviors for weak and strong interactions, respectively, in a single realization, at fixed magnetic field $B=3.35 \mathrm{~T}$ : At weak interactions $U \simeq 2 \mathrm{MHz}\left(U / \Delta \omega_{e} \simeq 0.04\right)$, the expectation value $\left\langle n\left|\hat{S}_{z}^{1}\right| n\right\rangle$ fluctuates between the fully polarized extremes $\pm 1 / 2$. In contrast, when the interactions dominate, $U \simeq$ $15,45 \mathrm{MHz},\left(U / \Delta \omega_{e} \simeq 0.3,0.8\right)$, the values of $\left\langle n\left|\hat{S}_{z}^{1}\right| n\right\rangle$ instead concentrate close to the equilibrium value 0 . This difference becomes sharper and sharper with increasing system size, as analyzed by the average variations of the local magnetization as a function of $N$, c.f. the right panel of Fig. 4. The extrapolation of such data to the thermodynamic limit allows one to locate the many-body localization transition. The data confirm that at strong interactions $U$ there is a thermal phase obeying ETH. This is exemplified by the expectation values of the local observable $S_{z}^{1}$ which coincide for all eigenstates and agree with the thermodynamic average. In contrast, for small $U$ and the accessible system sizes, we observe the fingerprints of many-body localization, with strong fluctuations of local observables between different eigenstates. In this case, thermodynamic expectation values can only be recovered by averaging over many eigenstates.

Internal thermalization among the electron spins explains the emergence of an effective spin temperature in DNP experiments, as long as the thermalization is much faster than the driving and bath relaxation processes. In the ergodic phase this is essentially always the case, given that the processes involving bath or drive are orders of magnitude slower than the internal spin dynamics.
Thermal eigenstates cannot encode any memory about local perturbations such as the frequency-selective spin flips driven by the microwave radiation. In contrast, in the localized phase of the isolated system, there exists an extensive set of local conserved quantities. This implies that two parameters $\beta_{s}$ and $h$ cannot contain sufficient information to describe local observables in all eigenstates of given total energy and polarization. As a consequence, the steady state and its properties will be more complex and depend on details of how the radiation and the bath couple to individual spins, and how those are coupled among each other. Our numerical results show that the steady state concentrates on eigenstates with a vanishing polarization of resonant electron spins and a strong polarization of the nonresonant ones.

The dependence of the nuclear spin polarization (via the spin temperature) on interaction strength and magnetic field, as shown in Fig. 2, can be understood, at least at a qualitative level. Indeed, when the ratio between interactions and magnetic field $U / B$ is increased, two competing effects are enhanced simultaneously within the thermal phase:

(1) The tendency towards thermalization increases, which eventually leads to ETH and the appearance of the spin temperature.

(2) The microwave irradiation is effective on a larger number of spins and thus acts less selectively. Indeed, all spins with Zeeman gap satisfying $\left|\omega_{e}+\Delta_{i}-\omega_{M W}\right|=1 / T_{2 e}^{\text {bath }}+$ $O(\delta E(U))$ will absorb the microwave irradiation efficiently. Hereby, $\delta E(U) \simeq \min \left[U^{2} / \Delta \omega_{e}, U\right]$ is the interaction-induced width of the local spectral functions of typical spins. The associated broadening of the absorption line implies a broader range of spins with suppressed polarization in the steady state and thus an increase in the resulting spin temperature.

Deep in the localized phase, local electron spin flips do not affect many other spins, so that only rarely a nuclear spin feels the impact of the microwave irradiation, and thus the average hyperpolarization is very low, as the only effect which can induce nuclear polarization is the solid effect. Upon increasing the interactions, more and more electron spins are coupled significantly with resonantly flipping spins, and thus the effect of the microwaves increases but still without a global spin temperature establishing. The maximal hyperpolarization is reached as delocalization occurs among the electron spins. At this point, all the electrons are involved in the transmission of polarization to the nuclei.

Thus, a sufficiently large $U$ is needed to ensure thermalization among the electron spins. However, making it too large broadens the absorption line and results in a stationary state with a higher spin temperature. Therefore, the inverse spin temperature, and thus the achieved hyperpolarization level of nuclear spins, reaches a maximum when $U / B$ is tuned to the proximity of the many-body localization transition: There, thermalization still occurs, but the microwave irradiation couples to spins in a maximally narrow frequency range, enabling a low spin temperature to emerge.

In Appendix D, we compare our present results with the phenomenological model proposed in Eq. (27). Using the value resulting from fit $C=0.0823$, we obtain perfect agreement with the numerical data in the regime where the spin temperature holds.

Figure 5 provides additional insight about the validity of the spin-temperature ansatz. It can be assessed using a standard 

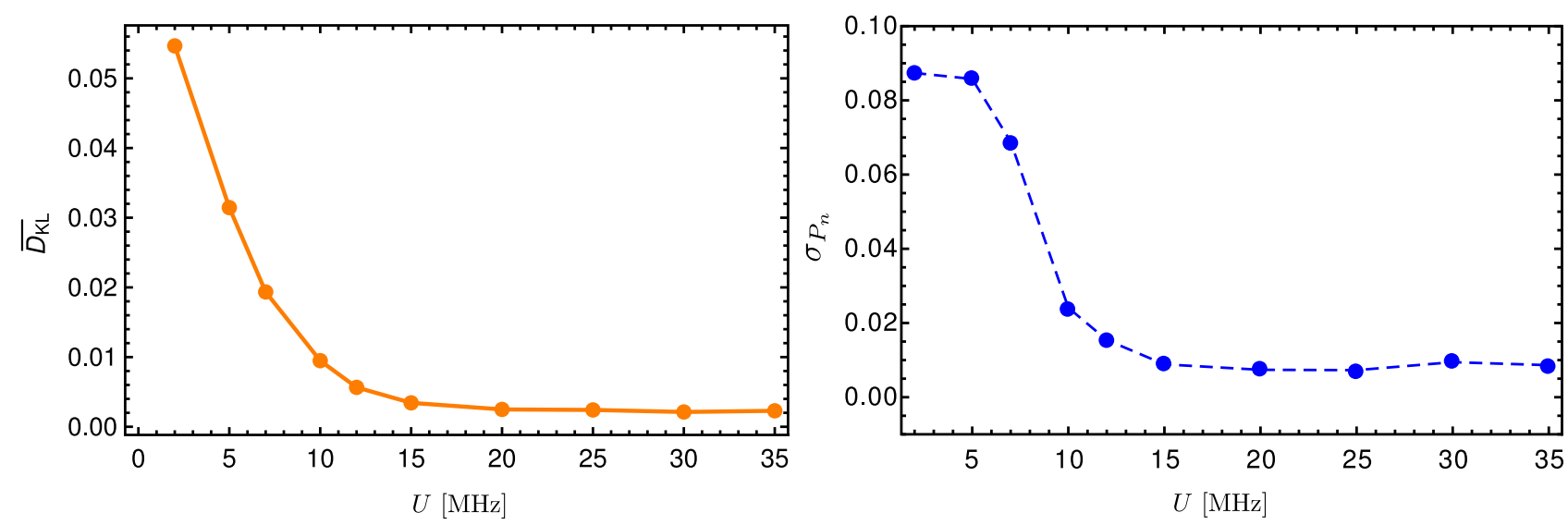

FIG. 5. (a) The Kullback-Leibler divergence, comparing the distribution in the stationary state $p_{n}^{\text {stat }}$ with the spin-temperature ansatz $p_{n}^{\text {Ans }}$ of (18). (b) The sample-to-sample fluctuations of the nuclear polarization as a function of $U$. The localized phase is characterized by vastly enhanced fluctuations of the polarization of the probing nuclear spin, demonstrating the absence of a homogeneous spin temperature in the electronic system.

statistical indicator, known as Kullback-Leibler divergence, which quantifies the information loss when $p_{n}^{\text {Ans }}$ is used to approximate $p_{n}^{\text {stat }}$. It is defined as

$$
D_{\mathrm{KL}}=\sum_{n} \ln \left(\frac{p_{n}^{\text {stat }}}{p_{n}^{\mathrm{Ans}}}\right) p_{n}^{\text {stat }} .
$$

The average of $D_{\mathrm{KL}}$ over disorder realizations is shown on the left of Fig. 5, as a function of the dipolar coupling. For small $U$, the large value of $D_{\mathrm{KL}}$ reflects the fact that the spintemperature ansatz does not capture the local behavior of the spin polarizations. At large $U$, where the spin-temperature picture applies, the value of $D_{\mathrm{KL}}$ becomes much smaller.

A fingerprint of the localization transition is seen in the sample-to-sample fluctuations of the stationary nuclear polarization shown on the right of Fig. 5. In the thermal phase, fluctuations originate only from finite-size fluctuations of the total energy and magnetization and are very small accordingly. In the localized phase instead, local processes control the nuclear polarization, whose stationary value depends on the presence or absence of few-body resonances involving the considered nuclear spin.

\section{CONCLUSION}

We presented a simple model for an improved description of DNP, which accounts for the crucial role played by dipolar interactions among radical spins. For sufficiently strong dipolar coupling, the electron spin system thermalizes internally and acts as an effective thermal bath for the nuclear spins and cools them down to the spin-temperature established among the electron spins. For this regime, we analytically estimated the achieved nuclear spin polarization in a perturbative expansion in the dipolar coupling, which we nevertheless assume strong enough to ensure a thermal phase.

In that phase the maximal hyperpolarization in DNP is obtained by minimizing the effective spin temperature. Our analysis shows that this is achieved by reducing the interaction strength to the maximal possible extent, that is by approaching the localization transition from the ergodic phase. This can be achieved by either reducing the radical concentration or increasing the magnetic field strength. The localized phase of the electron spins instead yields a significantly lower degree of nuclear spin polarization. Here a word of caution qualifying the meaning of "many-body localization" in real dipolar systems is in order. A genuine localization transition in the isolated system at finite energy density is expected only if the interactions are sufficiently short range. For dipolar interactions, already Anderson's work [6] suggested delocalization, even though on exponentially long timescales, as $U / \Delta \omega_{e}$ becomes small. This is because dipolar couplings decay as a marginal power law, which always allow spins to find resonant partners at large distance. Even in $d=2$ dimensions it has been argued that there are channels for delocalization [46-48], with, however, even longer timescales. When invoking a localization transition, we in fact allude to a strong crossover to very long thermalization timescales which grow exponentially with $\Delta \omega_{e} / U$. The latter makes the spin temperature ansatz break down rather quickly, too. Similarly, the mean field model is not expected to have a genuine localization transition as $N \rightarrow \infty$, which further justifies a posteriori our expansion around the noninteracting limit. Nevertheless, a thorough understanding of the thermalization processes and the involved timescales in the limit $N \rightarrow \infty$ require further analysis. It would be interesting to test these predictions in standard experimental DNP setups, both to validate our conclusions and to investigate the manifestation of the many-body localization transition in out-of-equilibrium stationary states.

It will be important to understand the crossover to a regime where the driving is eventually faster than the dephasing and/or the internal equilibration times, so that hole-burning phenomena and saturation of the driving efficiency become important. Furthermore, one should understand more thoroughly the localization transition as a function of the effective connectivity of the electron spins, which presumably varies with their dilution and the associated positional randomness in the radical spins. We plan to address these issues in a future publication.

To conclude, from a mere mathematical point of view, in an open system, the contact with the bath washes out any genuine 
nonanalyticity induced by many-body localization transition; nevertheless, we stress, as a general feature, that, when such a contact is sufficiently weak, manifestations of this remarkable effect are indeed observable in driven steady states.

\section{ACKNOWLEDGMENTS}

This work is supported by "Investissements d'Avenir" LabEx PALM (ANR-10-LABX-0039-PALM). We thank L. Mazza and Xiangyu Cao for interesting discussions, $\mathrm{S}$. Colombo Serra for the support with experimental data, and C. Zankoc for collaboration in the early stage of this work.

\section{APPENDIX A: BORGHINI MODEL WITH INTERACTIONS}

In this Appendix, we provide a general method to determine the two parameters $\beta_{s}, h$ conjugate to the two conserved quantities of the Hamiltonian $\hat{H}$ in (9). In the dynamics described by the master equation (16), the system exchanges simultaneously energy and magnetization every time a spin is flipped by the reservoir or the microwaves. We can compute explicitly the joint probability distribution $P(\Omega, S)$ of the energy and magnetization variation within a time interval $[t, t+\delta t]$ :

$$
\begin{aligned}
P(\Omega, S)= & \sum_{n n^{\prime}} \delta\left[\Omega-\left(\epsilon_{n}-\epsilon_{n^{\prime}}\right)\right] \delta\left[S-\left(s_{z}^{n}-s_{z}^{n^{\prime}}\right)\right] \\
& \times P_{n^{\prime} \rightarrow n}^{\delta t} p_{n^{\prime}}(t),
\end{aligned}
$$

where $P_{n^{\prime} \rightarrow n}^{\delta t}$ is the probability of passing from $n^{\prime} \rightarrow n$ during a time $\delta t$. In the limit $\delta t \rightarrow 0$, we therefore have $P_{n^{\prime} \rightarrow n}^{\delta t}=$ $W_{n^{\prime} \rightarrow n} \delta t$ for $n \neq n^{\prime}$, and $P_{n \rightarrow n}^{\delta t}=1-\sum_{n^{\prime} \neq n} P_{n \rightarrow n^{\prime}}=1-$ $\sum_{n^{\prime} \neq n} W_{n \rightarrow n^{\prime}} \delta t$. Substituting this into (A1), we arrive at $P(\Omega, S, t, \delta t)=P_{n \rightarrow n} \delta(\Omega) \delta(S)+\delta t p(\Omega, S, t)$ with

$$
\begin{aligned}
p(\Omega, S, t)= & \sum_{\substack{n, n^{\prime} \\
n \neq n^{\prime}}} \delta\left[\Omega-\left(\epsilon_{n}-\epsilon_{n^{\prime}}\right)\right] \delta\left[S-\left(s_{z}^{n}-s_{z}^{n^{\prime}}\right)\right] \\
& \times W_{n^{\prime} \rightarrow n} p_{n^{\prime}}(t) .
\end{aligned}
$$

Note that the term $P_{n \rightarrow n}$ does not contribute to the average of $\Omega$ and $S$. In the long time limit $p_{n}(t)$ tends to the stationary value $p_{n}^{\text {stat }}$. Using the rates in $(12,17)$ and the integral representation of the $\delta$ function, we have

$$
\begin{gathered}
\sum_{n \neq n^{\prime}} \delta\left[\Omega-\left(\epsilon_{n}-\epsilon_{n^{\prime}}\right)\right] \delta\left[S-\left(s_{z}^{n}-s_{z}^{n^{\prime}}\right)\right] W_{n^{\prime} \rightarrow n}^{\text {bath }} p_{n^{\prime}}^{\text {stat }} \\
=\sum_{j=1}^{N} \int \frac{d u}{2 \pi} \frac{d v}{2 \pi} e^{i u \Omega+i v S} \frac{4 h_{\beta}(-\Omega)}{T_{1 e}} \chi_{j j}(u, v),
\end{gathered}
$$

where $\chi_{j j}(u, v)$ is defined in (20) and we used rotational symmetry around the $z$ axis. Analogously, for the microwave rate we obtain

$$
\begin{aligned}
& \sum_{n \neq n^{\prime}} \delta\left[\Omega-\left(\epsilon_{n}-\epsilon_{n^{\prime}}\right)\right] \delta\left[S-\left(s_{z}^{n}-s_{z}^{n^{\prime}}\right)\right] W_{n^{\prime} \rightarrow n}^{\mathrm{MW}} p_{n^{\prime}}^{\text {stat }} \\
& \quad=\sum_{i j} \int \frac{d u}{2 \pi} \frac{d v}{2 \pi} e^{i u \Omega+i v S} \frac{4 \omega_{1}^{2} T_{2 e}^{\mathrm{bath}}}{1+\left(T_{2 e}^{\mathrm{bath}}\right)^{2}\left(\Omega-\omega_{\mathrm{MW}} S\right)^{2}} \chi_{i j}(u, v) .
\end{aligned}
$$

Note that in writing Eqs. (A3) and (A4), we used explicitly that $|n\rangle$ is simultaneously an eigenstate of $\hat{H}$ and $\hat{S}_{z}$. Thus, this derivation only holds for the conserved quantities of the model.

With the help of the Fourier transform of the correlation function

$$
\chi_{i j}(\Omega, S)=\int \frac{d u}{2 \pi} \frac{d v}{2 \pi} e^{i(\Omega u+S v)} \chi_{i j}(u, v),
$$

we can rewrite Eq. (A2) as

$$
\begin{aligned}
p_{\text {stat }}(\Omega, S)= & \frac{4 h_{\beta}(-\Omega)}{T_{1 e}} \sum_{j} \chi_{j j}(\Omega, S) \\
& +\frac{4 \omega_{1}^{2} T_{2 e}^{\text {bath }}}{1+\left(T_{2 e}^{\text {bath }}\right)^{2}\left(\Omega-\omega_{\mathrm{MW}} S\right)^{2}} \sum_{i j} \chi_{i j}(\Omega, S) .
\end{aligned}
$$

Imposing that the energy and magnetization flows vanish in the stationary state we find two equations

$$
\int d S d \Omega p_{\text {stat }}(\Omega, S) \Omega=0, \quad \int d S d \Omega p_{\text {stat }}(\Omega, S) S=0 .
$$

If we assume that the stationary state is effectively thermal, in agreement with (18), i.e., $p_{n}^{\text {stat }}=e^{-\beta_{s}\left(\epsilon_{n}+h s_{z, n}\right)} / Z$, Eqs. (A7) suffice to determine the two parameters $\beta_{s}, h$. The central ingredient, the correlation function $\chi_{i j}(u, v)$, is derived in the next Appendix.

\section{APPENDIX B: PERTURBATIVE CALCULATION OF THE CORRELATION FUNCTION IN THE MEAN-FIELD MODEL}

We now compute the correlation function $\chi_{i j}(u, v)$ to second order in the dipolar interaction strength $U$. It is analyzed in the mean-field model defined in (9), in the thermodynamic limit $N \rightarrow \infty$. We keep the inhomogeneities $\Delta_{i}$ finite and arbitrary. As the nuclear spin only slightly perturbs the electron spin Hamiltonian, we can neglect it in the estimation of $\beta_{s}$ and $h$ and focus on the subsystem of interacting electron spins:

$$
\begin{aligned}
\hat{H}_{e}(h) & =\sum_{i=1}^{N}\left(\omega_{e}+h+\Delta_{i}\right) \hat{S}_{z}^{i}+\sum_{i<j} U_{i j}\left(\hat{S}_{+}^{i} \hat{S}_{-}^{j}+\hat{S}_{-}^{i} \hat{S}_{+}^{j}\right) \\
& =\hat{H}_{0}+\hat{V}
\end{aligned}
$$

Here $\hat{H}_{0}$ is the noninteracting Hamiltonian, and $\hat{V}$ describes the couplings $U_{i j}$ between electron spins, which are taken to be Gaussian random variables with covariance matrix

$$
\overline{U_{i j} U_{k l}}=\frac{U^{2}}{N} \delta_{i k} \delta_{j l} \text { for } i<j, k<l .
$$

Note that, at order $O\left(U^{2}\right)$, upon averaging over $U_{i j}$, the correlation functions are diagonal in spin labels because of (B2), i.e., $\chi_{i j}(\Omega, S)=\delta_{i j} \chi_{j j}(\Omega, S)+o\left(U^{2}\right)$. Thus, we can restrict ourselves to the calculation of the dynamical correlation function $\chi_{j j}(u, v)$ of a single spin $j$. It can be simplified as

$$
\chi_{j j}(u, v)=\frac{e^{i(v-h u)} \Gamma_{+}^{j}(u, h)+e^{-i(v-h u)} \Gamma_{-}^{j}(-u, h)}{4},
$$


by introducing the $S_{+}^{j} S_{-}^{j}$ correlators

$$
\Gamma_{ \pm}^{j}(u, h)=Z^{-1} \operatorname{Tr}\left[e^{-\left(\beta_{s}-i u\right) \hat{H}_{e}(h)} \hat{S}_{ \pm}^{j} e^{-i u \hat{H}_{e}(h)} \hat{S}_{\mp}^{j}\right] .
$$

In the following we keep the dependence on $h$ implicit, since it simply amounts to changing $\omega_{e} \rightarrow \omega_{e}+h$ in $\hat{H}_{e}(0)$. In order to take advantage of the path integral formalism, we perform a Wick rotation and define $C_{ \pm}^{i}(\tau)=\Gamma_{ \pm}^{i}(-i \tau)$. From the cyclicity of the trace, we deduce $C_{-}^{i}(\tau)=C_{+}^{i}\left(\beta_{s}-\tau\right)$. We can thus restrict ourselves to calculating $C_{+}^{i}(\tau)$. We introduce the evolution operator in the interaction picture as $\hat{U}_{\tau}=e^{\tau \hat{H}_{0}} e^{-\tau \hat{H}}$, so that

$$
\frac{d \hat{U}_{\tau}}{d \tau}=\hat{H}_{0} \hat{U}_{\tau}-e^{\tau \hat{H}_{0}} \hat{H}_{e} e^{-\tau \hat{H}_{0}} \hat{U}_{\tau}=-\hat{V}_{I}(\tau) \hat{U}_{\tau},
$$

where $\hat{V}_{I}(\tau)=e^{\tau \hat{H}_{0}} \hat{V} e^{-\tau \hat{H}_{0}}$. Integrating this last equation over $[0, \tau]$ and re-injecting the resulting equation into itself, we obtain the second order expansion

$$
\begin{aligned}
e^{-\tau \hat{H}}= & e^{-\tau \hat{H}_{0}}-\int_{0}^{\tau} d t^{\prime} e^{\left(t^{\prime}-\tau\right) \hat{H}_{0}} \hat{V} e^{-t^{\prime} \hat{H}_{0}} \\
& +\int_{0}^{\tau} d t^{\prime} \int_{0}^{t^{\prime}} d t^{\prime \prime} e^{\left(t^{\prime}-\tau\right) \hat{H_{0}}} \hat{V} e^{\left(t^{\prime \prime}-t^{\prime}\right) \hat{H}_{0}} \hat{V} e^{-t^{\prime \prime} \hat{H}_{0}} .
\end{aligned}
$$

In order to keep track of all the terms in the expansion in $U$, we write $\hat{V} \rightarrow \epsilon \hat{V}$. We can expand the reduced partition function and the correlation function as

$$
\begin{gathered}
\frac{Z}{Z_{0}}=\left(1+\epsilon z^{(1)}+\epsilon^{2} z^{(2)}\right), \\
\frac{C_{+}^{i}(\tau) Z}{Z_{0}}=c_{+}^{i,(0)}(\tau)+\epsilon c_{+}^{i,(1)}(\tau)+\epsilon^{2} c_{+}^{i,(2)}(\tau),
\end{gathered}
$$

where $Z_{0}$ is the noninteracting $\left(U_{i j}=0\right)$ partition function. Then, we have the expansion

$$
\overline{C_{+}^{i}}=c_{+}^{i,(0)}(\tau)\left(1+\overline{\left(z^{(1)}\right)^{2}}-\overline{\left(z^{(2)}\right)}\right)-\overline{z^{(1)} c_{+}^{i,(1)}(\tau)}+\overline{c_{+}^{i,(2)}(\tau)},
$$

where the overline represents the average taken over the distribution of the couplings $U_{i j}$, at fixed values of the $\Delta_{i}$ 's. Since $\overline{\hat{V}}=0$ the first nonvanishing correction is quadratic. Moreover $z^{(1)}$ vanishes identically since $\left\langle n_{0}|\hat{V}| n_{0}\right\rangle=0$ for any eigenstate $\left|n_{0}\right\rangle$ of $\hat{H}_{0}$. After some algebra, we find the second order expression

$$
\overline{C_{+}^{i}(\tau)}=c_{+}^{i,(0)}(\tau)+\frac{U^{2}}{N} \sum_{l \neq i} \frac{d}{d \Delta_{i}}\left[\frac{c_{+}^{i,(0)}(\tau)-c_{+}^{l,(0)}(\tau)}{\Delta_{i}-\Delta_{l}}\right],
$$

where

$$
\begin{aligned}
c_{+}^{i,(0)}(\tau) & =\frac{\operatorname{Tr}\left[e^{-\left(\beta_{s}-\tau\right)\left(\omega_{e}+\Delta_{i}\right) S_{z}} S_{+}^{i} e^{-\tau\left(\omega_{e}+\Delta_{i}\right) S_{z}} S_{-}^{i}\right]}{\operatorname{Tr}\left[e^{-\beta_{s}\left(\omega_{e}+\Delta_{i}\right) S_{z}}\right]} \\
& =\frac{e^{\tau\left(\omega_{e}+\Delta_{i}\right)}}{1+e^{\beta_{s}\left(\omega_{e}+\Delta_{i}\right)}} .
\end{aligned}
$$

A simpler way to derive Eq. (B10) is to note, by simple power counting, that at order $U^{2}$, the perturbation in $\overline{C_{+}^{i}(\tau)}$ must consist in additive contributions from all spins $l \neq i$. The term inside the sum in (B10) can then be obtained by solving exactly the case $N=2$ for an arbitrary value of the coupling $U_{12}$ in (B1) and expanding up to the order $U_{12}^{2}$. Note that the expression (B11) allows us also to compute the local polarizations:

$$
\begin{aligned}
P_{i} & =2 \operatorname{Tr}\left[S_{z}^{i} \rho\right]=\operatorname{Tr}\left[S_{+}^{i} S_{-}^{i} \rho\right]-\operatorname{Tr}\left[S_{-}^{i} S_{+}^{i} \rho\right] \\
& =\overline{C_{+}^{i}(0)}-\overline{C_{-}^{i}(0)}=P_{i}^{0}+\frac{U^{2}}{N} \sum_{l \neq i} \frac{d}{d \Delta_{i}}\left[\frac{P_{i}^{0}-P_{l}^{0}}{\Delta_{i}-\Delta_{l}}\right],
\end{aligned}
$$

where $P_{i}^{0}=-\tanh \left(\frac{\beta_{s}\left(\omega_{e}+\Delta_{i}\right)}{2}\right)$ are the polarizations in the noninteracting limit $U=0$.

Performing back the Wick rotation $\tau=i u$ and replacing $\omega_{e} \rightarrow \omega_{e}+h$ in (B10) and (B11), we obtain $\Gamma_{ \pm}^{i}(u, h)$

$$
\begin{aligned}
\Gamma_{+}^{i}(u, h)= & \left.\frac{e^{i u\left(\omega_{e}+h+\Delta_{i}\right)}}{1+e^{\beta_{s}\left(\omega_{e}+h+\Delta_{i}\right)}}\right] \\
& +\frac{U^{2}}{N} \sum_{l \neq i} \frac{d}{d \Delta_{i}}\left[\frac{\frac{e^{i u\left(\omega_{e}+h+\Delta_{i}\right)}}{1+e^{\beta_{s}\left(\omega_{e}+h+\Delta_{i}\right)}}-\frac{e^{i u\left(\omega_{e}+h+\Delta_{l}\right)}}{1+e^{\beta_{s}\left(\omega_{e}+h+\Delta_{l}\right)}}}{\Delta_{i}-\Delta_{l}}\right], \\
\Gamma_{-}^{i}(u, h)= & \left.\frac{e^{\left(\beta_{s}-i u\right)\left(\omega_{e}+h+\Delta_{i}\right)}}{1+e^{\beta_{s}\left(\omega_{e}+h+\Delta_{i}\right)}}\right] \\
& +\frac{U^{2}}{N} \sum_{l \neq i} \frac{d}{d \Delta_{i}}\left[\frac{\frac{e^{\left(\beta_{s}-i u\right)\left(\omega_{e}+h+\Delta_{i}\right)}}{1+e^{\beta_{s}\left(\omega_{e}+h+\Delta_{i}\right)}}-\frac{e^{\left(\beta_{s}-i u\right)\left(\omega_{e}+h+\Delta_{l}\right)}}{1+e^{\beta_{s}\left(\omega_{e}+h+\Delta_{l}\right)}}}{\Delta_{i}-\Delta_{l}}\right] .
\end{aligned}
$$

\section{APPENDIX C: EQUATIONS FOR $\beta_{s}$ AND $h$ IN THE MEAN-FIELD MODEL}

Using (B11) and (A5), the correlation $\chi_{j j}^{U=0}(\Omega, S)$ in the noninteracting case is:

$$
\begin{aligned}
\chi_{j j}^{U=0}(\Omega, S)= & \frac{\delta(S-1) h_{\beta_{s}}\left(\omega_{e}+h+\Delta_{j}\right) \delta\left(\Delta_{j}-\Omega+\omega_{e}\right)}{4} \\
& +\frac{\delta(S+1) h_{\beta_{s}}\left(-\omega_{e}-h-\Delta_{j}\right) \delta\left(\Delta_{j}+\Omega+\omega_{e}\right)}{4} .
\end{aligned}
$$

Using (A6) and (A7), the integration over $S$ and $\Omega$ leads to the equations which fix $h$ and $\beta_{s}$ of the spin temperature ansatz:

$$
\begin{gathered}
\sum_{j} \kappa\left(\omega_{e}+\Delta_{j}\right)=0, \\
\sum_{j}\left(\omega_{e}+\Delta_{j}\right) \kappa\left(\omega_{e}+\Delta_{j}\right)=0,
\end{gathered}
$$

where $\kappa(\omega)$ is defined in (22). Equations (21) are recovered in the large $N$ limit, where the sums over $j$ can be converted into integrals over the distribution of inhomogeneities, $f(\Delta)$. Then, since (B10) is a linear combination of the functions the $c_{+}^{i,(0)}(\tau)$, to order $U^{2}$ the equations simply become

$$
\begin{aligned}
\sum_{j}\left[\kappa\left(\omega_{e}+\Delta_{j}\right)+\frac{U^{2}}{N} \sum_{l \neq j} \frac{d}{d \Delta_{j}}\right. \\
\left.\left(\frac{\kappa\left(\omega_{e}+\Delta_{j}\right)-\kappa\left(\omega_{e}+\Delta_{l}\right)}{\Delta_{j}-\Delta_{l}}\right)\right]=0,
\end{aligned}
$$




$$
\begin{aligned}
& \sum_{j}\left[\left(\omega_{e}+\Delta_{j}\right) \kappa\left(\omega_{e}+\Delta_{j}\right)+\frac{U^{2}}{N} \sum_{l \neq j} \frac{d}{d \Delta_{j}}\right. \\
& \left.\left(\frac{\left(\omega_{e}+\Delta_{j}\right) \kappa\left(\omega_{e}+\Delta_{j}\right)-\left(\omega_{e}+\Delta_{l}\right) \kappa\left(\omega_{e}+\Delta_{l}\right)}{\Delta_{j}-\Delta_{l}}\right)\right]=0,
\end{aligned}
$$

the integral version of which is given in Eqs. (24).

\section{APPENDIX D: PHENOMENOLOGICAL MODELS FOR THE SPIN TEMPERATURE-BORGHINI'S MODEL AND AN IMPROVED VERSION}

A first phenomenological approach to the spin temperature, as observed in dynamic nuclear polarization, was introduced in the sixties by Borghini [32] and later reviewed by Abragam and Goldman [49]. They consider electron spins with an inhomogeneous broadening of the Zeeman splitting given by the normalized distribution $f(\omega)$ with mean

$$
\int d \omega f(\omega) \omega=\omega_{e} .
$$

Two ingredients are taken into account: the irradiation by the microwave field, that tries to saturate the electrons resonating at its frequency, and the relaxation by coupling to the lattice at temperature $\beta^{-1}$. Borghini obtained equations expressing the conservation of the total magnetization and energy in Eqs. (21), with $\kappa(\omega)$ given by Eq. (23). Borghini assumed strong microwave irradiation, postulating that the electrons resonating exactly at $\omega_{\mathrm{MW}}$ are fully saturated. This forces $h=\omega_{\mathrm{MW}}$ in the quasiequilibrium ansatz for the electron polarization, and leaves only the spin temperature $\beta_{s}^{-1}$ as parameter,

$$
P_{e}(\omega)=-\tanh \left(\frac{\beta_{s}}{2}\left(\omega-\omega_{\mathrm{MW}}\right)\right) .
$$

With this assumption, Eqs. (21) can be transformed into a single equation by multiplying Eq. (21b) by $\omega_{\mathrm{MW}}$ and subtracting Eq. (21a). This leads to the condition

$$
\int d \omega f(\omega)\left(\omega-\omega_{\mathrm{MW}}\right) P_{e}(\omega)=\left(\omega_{e}-\omega_{\mathrm{MW}}\right) P_{0},
$$

where $P_{0}$ is the electron polarization at equilibrium with the bath, $P_{0}=-\tanh \left(\beta \omega_{e} / 2\right)$. We neglected its weak dependence on $\omega \simeq \omega_{e}$, as the average electron Zeeman gap $\omega_{e}$ is typically three orders of magnitude larger than its broadening $\omega-\omega_{e} \simeq$ $\Delta \omega_{e}$. The solution of this equation provides a value of the spin temperature and suggests an optimal irradiation frequency $\omega_{\mathrm{MW}}$.

We see from Eqs. (D2) and (D3) that the parameter $\beta_{s}$ has an interesting property: If one irradiates at $\omega_{\mathrm{MW}}<\omega_{e}$, one needs $\beta_{s}>0$ in order to verify Eq. (D3). On the other hand, irradiating at $\omega_{\mathrm{MW}}>\omega_{e}$, we need a negative value $\beta_{s}<0$. For irradiation precisely at the electron resonance, $\omega_{\mathrm{MW}}=\omega_{e} \beta_{s}=0$ results. Additionally, if the distribution function $f(\omega)$ is symmetric around $\omega_{e}$, the spin temperature is an odd function of $\omega_{e}-\omega_{\mathrm{MW}}$. This property holds beyond Borghini's approximations. Indeed, it is exactly verified by the DNP profile for our model of Sec. II B (see Fig. 3). In reality, the symmetry of $f(\omega)$ is only approximate, but the spin temperature still exhibits the change of sign described above.

Negative temperatures arise in equilibrium situations of systems with bounded local spectrum, where high energy modes are more populated than low energy ones, and energy conservation does not allow us to redistribute the excess energy to revert to a usual distribution. In our case, this situation arises naturally when the irradiation frequency lies above $\omega_{e}$, so that energy is fed predominantly into the higher energy modes. Recently, such an effect has been used to suppress the fluctuations in resonant absorption with possible application in quantum information processing [50]. We plan to investigate similar phenomena in our system in future work.

The Borghini model yields a reasonable description of the spin-temperature regime. Nevertheless, it has some limitations and pathologies, namely:

(1) It overestimates the value of $\beta_{s}$, which translates in unrealistic high values of the nuclear polarization.

(2) It predicts no dependence of $\beta_{s}$ on the microscopic parameters of the system, e.g., the electron concentration (or electron interaction strength $U), T_{2 e}^{\text {bath }}$, the microwave intensity $\omega_{1}$, etc.

A significant improvement of this model is achieved by considering that the absorption line for the microwave irradiation is not infinitely sharp, but a Lorentzian, as described by the expression $\kappa(\omega)$ in Eq. (22). This yields the estimate for the nuclear polarization represented by the dashed line in Fig. 2 for $U=0$. A further phenomenological improvement is obtained by realizing that the width of the Lorentzian should reflect the dephasing time of single spins, rather than eigenstates. This $T_{2 e}$ is affected by dipolar couplings of a given spin with other spins. An estimate of spin flip rates based on Fermi's golden rule suggests the interaction dependent single-spin dephasing rate

$$
\frac{1}{T_{2 e}(U)}=\frac{1}{T_{2 e}^{\text {bath }}}+C \frac{U^{2}}{\Delta \omega_{e}},
$$

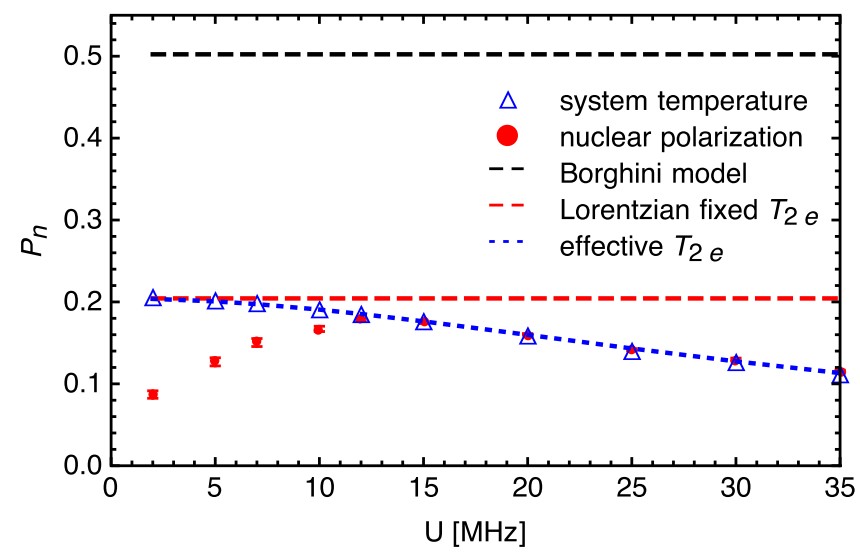

FIG. 6. Comparison between Borghini's original model and our improved phenomenological model based on a $U$-dependent decoherence time $T_{2 e}(U)$. The two models are compared with the numerical data obtained by exact diagonalization. While Borghini's prediction strongly overestimates the nuclear polarization, the improved model yields very good agreement with the numerical data. 
where $\Delta \omega_{e}$ is the disorder on the electron Zeeman frequency given in Table I. Here $C$ is a numerical constant that we determine to best fit the numerical data for the observed spin temperature. The value of $C$ depends on the characteristics of the system. In contrast to the perturbative expansion, which gives reliable predictions only for small $U$, the above phenomenological model captures well an important aspect of the interactions even at moderately strong interactions.

In Fig. 6, we show the predictions for the nuclear polarization, obtained from Borghini's original model and the model with Lorentzian absorption lines governed by the effective $T_{2 e}(U)$ of Eq. (D4). They are compared to the numerical results of Fig. 2. Note that we plot both the simulated nuclear polarization and the result obtained from the fitting method for the spin temperature. As mentioned before, Borghini's original model of Eq. (D3) predicts an extremely high nuclear polarization of $50 \%$ without any dependence on the interaction strength. In contrast, our phenomenological model with dephasing time $T_{2 e}(U)$ yields an extremely good agreement with numerical data, with only a single free fit parameter.
[1] M. Rigol, V. Dunjko, and M. Olshanii, Nature (London) 452, 854 (2008)

[2] A. Polkovnikov, K. Sengupta, A. Silva, and M. Vengalattore, Rev. Mod. Phys. 83, 863 (2011).

[3] J. M. Deutsch, Phys. Rev. A 43, 2046 (1991); M. Srednicki, Phys. Rev. E 50, 888 (1994).

[4] A. De Luca and A. Scardicchio, Europhys. Lett. 101, 37003 (2013).

[5] A. De Luca, B. L. Altshuler, V. E. Kravtsov, and A. Scardicchio, Phys. Rev. Lett. 113, 046806 (2014).

[6] P. W. Anderson, Phys. Rev. 109, 1492 (1958).

[7] R. Nandkishore and D. A. Huse, Annu. Rev. Condens. Matter Phys. 6, 15 (2015).

[8] I. V. Gornyi, A. D. Mirlin, and D. G. Polyakov, Phys. Rev. Lett. 95, 206603 (2005).

[9] D. Basko, I. Aleiner, and B. Altshuler, Ann. Phys. 321, 1126 (2006).

[10] A. Pal and D. A. Huse, Phys. Rev. B 82, 174411 (2010).

[11] J. H. Bardarson, F. Pollmann, and J. E. Moore, Phys. Rev. Lett. 109, 017202 (2012).

[12] R. Vosk and E. Altman, Phys. Rev. Lett. 110, 067204 (2013).

[13] D. A. Huse, R. Nandkishore, and V. Oganesyan, Phys. Rev. B 90, 174202 (2014).

[14] M. Serbyn, Z. Papić, and D. A. Abanin, Phys. Rev. Lett. 111, 127201 (2013).

[15] V. Ros, M. Müller, and A. Scardicchio, Nucl. Phys. B 891, 420 (2015).

[16] J. Z. Imbrie, J. Stat. Phys. 163, 998 (2016).

[17] B. Bauer and C. Nayak, J. Stat. Mech. (2013) P09005.

[18] A. Chandran, I. H. Kim, G. Vidal, and D. A. Abanin, Phys. Rev. B 91, 085425 (2015).

[19] M. Schreiber, S. S. Hodgman, P. Bordia, H. P. Lüschen, M. H. Fischer, R. Vosk, E. Altman, U. Schneider, and I. Bloch, Science 349, 842 (2015).

[20] J. Smith, A. Lee, P. Richerme, B. Neyenhuis, P. W. Hess, P. Hauke, M. Heyl, D. A. Huse, and C. Monroe, Nat. Phys. (2016), doi:10.1038/nphys3783.

[21] S. Ghosh, R. Parthasarathy, T. Rosenbaum, and G. Aeppli, Science 296, 2195 (2002).

[22] G. Feher, Phys. Rev. 114, 1219 (1959).

[23] M. Serbyn, Z. Papić, and D. A. Abanin, Phys. Rev. Lett. 110, 260601 (2013).

[24] A. Chandran, V. Khemani, C. R. Laumann, and S. L. Sondhi, Phys. Rev. B 89, 144201 (2014).

[25] A. Abragam and M. Goldman, Nuclear Magnetism: Order and Disorder (Oxford University Press, Oxford, UK, 1982).
[26] Empirically a glassy atomic structure is necessary to obtain significant hyperpolarization. The reason is not entirely established, but such a structure presumably helps to ensure a homogeneous dilution of radicals (and to prevent the clustering of radicals at lower-dimensional grain boundaries).

[27] J. H. Ardenkjær-Larsen, B. Fridlund, A. Gram, G. Hansson, L. Hansson, M. H. Lercrhe, R. Servin, M. Thaning, and K. Golman, Proc. Natl. Acad. Sci. USA 100, 10158 (2003).

[28] K. Golman, M. Lerche, R. Pehrson, J. H. Ardenkjaer-Larsen et al., Cancer Res. 66, 10855 (2006).

[29] B. Provotorov, Zh. Eksp. Teor. Fiz. 41, 1582 (1962) [JETP 14, 1126 (1962)].

[30] L. Lumata, A. K. Jindal, M. E. Merritt, C. R. Malloy, A. D. Sherry, and Z. Kovacs, J. Am. Chem. Soc. 133, 8673 (2011).

[31] F. Kurdzesau, B. van den Brandt, A. Comment, P. Hautle, S. Jannin, J. van der Klink, and J. Konter, J. Phys. D: Appl. Phys. 41, 155506 (2008).

[32] M. Borghini, Phys. Rev. Lett. 20, 419 (1968).

[33] A. De Luca and A. Rosso, Phys. Rev. Lett. 115, 080401 (2015).

[34] Y. Hovav, A. Feintuch, and S. Vega, J. Magn. Reson. 207, 176 (2010); 214, 29 (2012); Phys. Chem. Chem. Phys. 15, 188 (2013).

[35] A. Karabanov, D. Wiśniewski, I. Lesanovsky, and W. Köckenberger, Phys. Rev. Lett. 115, 020404 (2015).

[36] S. Colombo Serra, M. Filibian, P. Carretta, A. Rosso, and F. Tedoldi, Phys. Chem. Chem. Phys. 16, 753 (2014).

[37] F. Buccheri, A. De Luca, and A. Scardicchio, Phys. Rev. B 84, 094203 (2011).

[38] Explicitly, the energy shifts are chosen as $\Delta_{i}=\Delta \omega_{e}\left(\frac{2 i-N-1}{N}\right)$ with $i=1, \ldots, N$.

[39] M. Filibian, S. C. Serra, M. Moscardini, A. Rosso, F. Tedoldi, and P. Carretta, Phys. Chem. Chem. Phys. 16, 27025 (2014).

[40] F. Petruccione and H.-P. Breuer, The Theory of Open Quantum Systems (Oxford University Press, Oxford, UK, 2002).

[41] H. Jóhannesson, S. Macholl, and J. H. Ardenkjaer-Larsen, J. Magn. Reson. 197, 167 (2009).

[42] Indeed, the electron spin flips induced by the bath are the slowest process and fully determine the polarization time, $T_{\text {pol }}=\frac{N_{n}}{N_{e}} T_{1 e}[39]$.

[43] S. C. Serra, A. Rosso, and F. Tedoldi, Phys. Chem. Chem. Phys. 14, 13299 (2012).

[44] S. Serra Colombo, A. Rosso, and F. Tedoldi, Phys. Chem. Chem. Phys. 15, 8416 (2013).

[45] A. Karabanov, A. van der Drift, L. J. Edwards, I. Kuprov, and W. Köckenberger, Phys. Chem. Chem. Phys. 14, 2658 (2012).

[46] A. L. Burin, arXiv:cond-mat/0611387. 
[47] N. Y. Yao, C. R. Laumann, S. Gopalakrishnan, M. Knap, M. Müeller, E. A. Demler, and M. D. Lukin, Phys. Rev. Lett. 113, 243002 (2014).

[48] D. Gutman, I. Protopopov, A. Burin, I. Gornyi, R. Santos, and A. Mirlin, Phys. Rev. B 93, 245427 (2016).
[49] A. Abragam and M. Goldman, Rep. Prog. Phys. 41, 395 (1978).

[50] C. Latta, A. Hogele, Y. Zhao, A. N. Vamivakas, P. Maletinsky, M. Kroner, J. Dreiser, I. Carusotto, A. Badolato, D. Schuh, W. Wegscheider, M. Atature, and A. Imamoglu, Nat. Phys. 5, 758 (2009). 